\title{
TRIVIALITY OF VECTOR BUNDLES ON SUFFICIENTLY TWISTED IND-GRASSMANNIANS
}

\author{
IVAN PENKOV AND ALEXANDER S. TIKHOMIROV
}

\begin{abstract}
Twisted ind-Grassmannians are ind-varieties $\mathbf{G}$ obtained as direct limits of Grassmannians $G\left(r_{m}, V^{r_{m}}\right)$, for $m \in \mathbf{Z}_{>0}$, under embeddings $\varphi_{m}: G\left(r_{m}, V^{r_{m}}\right) \rightarrow G\left(r_{m+1}, V^{r_{m+1}}\right)$ of degree greater than one. It has been conjectured in [PT] and [DP] that any vector bundle of finite rank on a twisted ind-Grassmannian is trivial. We prove this conjecture under the assumption that the ind-Grassmannian $\mathbf{G}$ is sufficiently twisted, i.e. that $\lim _{m \rightarrow \infty} \frac{r_{m}}{\operatorname{deg} \varphi_{1} \ldots \operatorname{deg} \varphi_{m}}=0$. 2000 Mathematics Subject Classification, Primary 14M15, (Secondary 14J60, 32L05).
\end{abstract}

\section{INTRODUCTION}

Ind-Grassmannians are ind-varieties defined by chains of embeddings

$$
G\left(r_{1}, V^{n_{1}}\right) \stackrel{\varphi_{1}}{\longleftrightarrow} G\left(r_{2}, V^{n_{2}}\right) \stackrel{\varphi_{2}}{\longrightarrow} \ldots \stackrel{\varphi_{m-1}}{\longrightarrow} G\left(r_{m}, V^{n_{m}}\right) \stackrel{\varphi_{m}}{\longrightarrow} \ldots
$$

where $G(r, V)$ denotes the Grassmanian of $r$-planes in a finite dimensional vector space $V$. Any of the embeddings $\varphi_{m}$ has a well defined degree $\operatorname{deg} \varphi_{m}$, and the ind-Grassmannian defined by (11) is twisted if $\operatorname{deg} \varphi_{m}>m$ for infinitely many indices. In the special case when $r_{m}=1$ and $\operatorname{deg} \varphi_{m}=1$ for all $m$, the study of finite rank vector bundles on ind-Grassmannians goes back to W.Barth, A.Van de Ven and A.N.Tyurin, BV], T]. In this case the ind-Grassmannian is just the infinite projective space $\mathbb{P}^{\infty}$, and the remarkable Barth-Van de Ven-Tyurin Theorem claims that any vector bundle of finite rank on $\mathbb{P}^{\infty}$ is isomorphic to a direct sum of line bundles. Historically, this is the first manifestation of a general phenomenon that seems to take place for ind-varieties defined via sequences of embeddings similar to (1) with $G\left(r_{m}, V_{m}\right)$ replaced by arbitrary compact homogeneous spaces: in all cases known, the restriction of any finite rank vector bundle on the ind-variety to a large enough finite dimensional homogeneous subspace is equivariant. Around the same time this phenomenon occured also in the important work of E. Sato who gave an independent proof of the Barth-Van de Ven-Tyurin Theorem, S1. Shortly after that Sato established a more general result which applies in particular to the ind-Grassmannian $\mathbf{G}(r, V)$ of $r$-planes in a countable dimensional vector space $V$, [S2].

More recently the subject has been revisited in the papers [DP], [CT] and [PT]. In particular, in [PT] a general conjecture about finite rank vector bundles on ind-Grassmannians has been stated. In fact, as we show in [PT], if the ind-Grassmannian is not twisted (which is easily seen to be equivalent to assuming that $\operatorname{deg} \varphi_{m}=1$ for all $m$ ), this conjecture is a relatively straightforward corollary of Sato's result. This leaves open the case of a twisted ind-Grassmannian, in which case the conjecture claims simply that finite rank vector bundle on such an ind-Grassmannian is trivial. So far this latter conjecture is established in the following three cases: for a rank two bundle on any twisted ind-Grassmannian [PT], for any finite rank bundle on any twisted projective ind-space (a twisted projective ind-space can be defined via the sequence (1) where $r_{m}=1$ and $\operatorname{deg} \varphi_{m}>1$ for all $m$ ) [DP and for an arbitrary finite rank bundle on some special twisted ind-Grassmannians (here $\varphi_{m}$ are twisted extensions as defined in $[\mathrm{DP}]$ ).

In the present paper we consider the case of arbitrary finite rank vector bundle on arbirary twisted ind-Grassmannians satisfying the condition $\operatorname{dim} r_{m}=$ const for all $m$. In fact, we work with a more general class of twisted ind-Grassmannians which we call sufficiently twisted. They 
are defined via the condition

$$
\lim _{m \rightarrow \infty} \frac{r_{m}}{\operatorname{deg} \varphi_{1} \ldots \operatorname{deg} \varphi_{m-1}}=0
$$

Our main idea is that the relatively simple proof of the conjecture in the case of a twisted ind-projective space, [DP], admits an interesting generalization. More precisely, the original method is based on the study of certain morphisms of $\mathbb{P}^{1} \times \mathbb{P}^{1}$ into larger and larger projective spaces. Under the assumption that a twisted infinite projective space admits a non-trivial vector bundle, one pulls it back to $\mathbb{P}^{1} \times \mathbb{P}^{1}$, and for a sufficiently large projective space the pullback is forced to have numerical invariants which yield a contradiction. The main technical achievement of the present paper is the introduction of an appropriate class of maps of $\mathbb{P}^{1} \times \mathbb{P}^{1}$ into a twisted ind-Grassmannian (defined in terms of a construction of a certain vector bundles on $\mathbb{P}^{1} \times \mathbb{P}^{1}$, see Section 5 below) and the corresponding extension of certain estimates in [DP] concerning lines in $\mathbb{P}^{n}$ to Segre curves of degree $2 r$ in $G(r, V)$. The only limitation of this more general method has to do with fact that it is still based on the specific properties of the surface $\mathbb{P}^{1} \times \mathbb{P}^{1}$. This explains the condition (2).

Acknowledgement. We acknowledge the support and hospitality of the Max Planck Institute for Mathematics in Bonn where the present paper was conceived. A. S. T. also acknowledges partial support from Jacobs University Bremen.

\section{Notation And Conventions}

Our notation is mostly standard. The ground field is $\mathbb{C}$. All vector bundles considered are assumed to have finite rank. We do not make a distinction between locally free sheaves of finite rank and vector bundles. If $\mathcal{F}$ is a sheaf of $\mathcal{O}_{X}$-modules on an algebraic variety $X$, $\mathcal{F}^{n}$ denotes the direct sum of $n$ copies of $\mathcal{F}, H^{i}(\mathcal{F})$ denotes the $i^{\text {th }}$ cohomology group of $\mathcal{F}$, $h^{i}(\mathcal{F}):=\operatorname{dim} H^{i}(\mathcal{F})$, and $\mathcal{F}^{\vee}$ stands for the dual bundle, i. e. $\mathcal{F}^{\vee}:=\mathcal{H} m_{\mathcal{O}_{X}}\left(\mathcal{F}, \mathcal{O}_{X}\right)$. If $Z \subset X$ is a subvariety, $I_{Z, X}$ denotes the sheaf of ideals corresponding to $Z$.

By $G(r, V)$ we denote the Grassmannian of $r$-dimensional subspaces of a vector space $V$; unless the contrary is stated explicitly, we assume that $\operatorname{dim} V<\infty, r \neq 1, r \neq \operatorname{dim} V-1$.

By a rational curve we always mean a curve isomorphic to $\mathbb{P}^{1}$, i. e. for convenience we assume a rational curve to be smooth. If $C$ is a rational curve, $\mathcal{O}_{C}(i)$ stands for a line bundle on $C$ with first Chern class equal to $i \in \mathbf{Z}$. A line in $G(r, V)$ is a rational curve of degree 1 and is determined by a flag of $V_{1} \subset V_{2}$ of subspaces in $V$ with $\operatorname{dim} V_{1}=r-1, \operatorname{dim} V_{2}=r+1$.

If $C \subset X$ is a rational curve in an algebraic variety $X$ and $E$ is a vector bundle on $X$, then by a classical theorem of Grothendieck, $E_{\mid C}$ is isomorphic to $\bigoplus_{i} \mathcal{O}_{C}\left(d_{i}\right)$ for some $d_{1} \geq d_{2} \geq$ $\cdots \geq d_{\mathrm{rk} E}$. We call the ordered $\mathrm{rkE}$-tuple $\left(d_{1}, \ldots, d_{\mathrm{rk} E}\right)$ the splitting type of $E_{\mid C}$ and denote it by $\mathbf{d}_{E}(C)$.

We call a curve $C=\bigcup_{i=1}^{r} C_{i}$, where $C_{i}$ are rational curves, a chain of rational curves, if, for each $i<r$, the intersection $C_{i} \cap C_{i+1}$ is a transversal intersection at a single point and there are no other intersections of the curves $C_{i}$. If $C$ is a chain of rational curves, $\mathcal{O}_{C}\left(n_{1}, \ldots, n_{r}\right)$ denotes a line bundle on $C$ such that $\mathcal{O}_{C}\left(n_{1}, \ldots, n_{r}\right)_{\mid C_{i}} \simeq \mathcal{O}_{C_{i}}\left(n_{i}\right)$.

Finally, under a partition of $n \in \mathbf{Z}_{>0}$ (respectively, a strict partition of $n$ ) we understand a $k$-tuple $\left(n_{1}, \ldots, n_{k}\right) \in \mathbf{Z}_{\geq 0}^{k}$ (respectively, $\left.\left(n_{1}, \ldots, n_{k}\right) \in \mathbf{Z}_{>0}^{k}\right)$ with $\sum_{i=1}^{k} n_{i}=n$.

\section{An estimate for $\mathbf{D}_{E}(C)$}

For a vector bundle $E_{C}$ on a rational curve $C$ with splitting type $\mathbf{d}\left(E_{C}\right)=$ $\left(\mathbf{d}_{1}\left(E_{C}\right), \ldots, \mathbf{d}_{\mathrm{rk} E}\left(E_{C}\right)\right)$, set $\mathbf{D}\left(E_{C}\right):=\mathbf{d}_{1}\left(E_{C}\right)-\mathbf{d}_{\mathrm{rk} E}\left(E_{C}\right)$. Our objective in this section is to prove the following theorem. 
Theorem 3.1. Let $\pi: X \rightarrow B$ be a flat family whose fibers are chains of rational curves and whose generic fiber is a rational curve. Assume that, for a point $0 \in B, C_{0}:=\pi^{-1}(0)$ is a chain of rational curves $C_{1} \cup \ldots \cup C_{r}, r \geq 1$. Let $E$ be a vector bundle on $X$. Then there exists a neighbourhood $U$ of the point 0 in $B$ such that for any $t \in U$ for which $C_{t}=\pi^{-1}(t)$ is a rational curve, one has

$$
\mathbf{D}\left(E_{\mid C_{t}}\right) \leq \sum_{i=1}^{r} \mathbf{D}\left(E_{\mid C_{i}}\right) .
$$

Proof. The proof is based on two auxiliary results, namely Corollary 3.4 and Lemma 3.5, which we prove later on in this section. First, Corollary 3.4 implies that for any integers $n_{1}, \ldots, n_{r}$ there exists a neighbourhood $U^{\prime} \subset B$ of the point 0 and a line bundle $\mathcal{L}$ on $\pi^{-1}\left(U^{\prime}\right)$ such that $\mathcal{L}_{\mid C_{0}} \simeq \mathcal{O}_{C_{0}}\left(n_{1}, \ldots, n_{r}\right)$. Hence $E_{\mid C_{0}} \otimes \mathcal{L} \simeq E_{\mid C_{0}}\left(n_{1}, \ldots, n_{r}\right)$ and $E_{\mid C_{t}} \otimes \mathcal{L} \simeq E_{\mid C_{t}}\left(n_{1}+\ldots+n_{r}\right)$ for any $t \in U^{\prime}$ for which $C_{t}=\pi^{-1}(t)$ is a rational curve. By semicontinuity,

$$
h^{0}\left(\left(E_{\mid C_{0}}\right)\left(n_{1}, \ldots, n_{r}\right)\right) \geq h^{0}\left(\left(E_{\mid C_{t}}\right)\left(n_{1}+\ldots+n_{r}\right)\right) .
$$

Therefore, for $n_{i}=-\mathbf{d}_{1}\left(E_{\mid C_{i}}\right)-\delta_{i}$, where $\delta_{i}$ are as in Lemma 3.5, the inequality (44) and Lemma 3.5 imply

$$
h^{0}\left(E_{\mid C_{t}}\left(-\sum_{i=1}^{r} \mathbf{d}_{1}\left(E_{\mid C_{i}}\right)-\sum_{i=1}^{r} \delta_{i}\right)\right)=0 .
$$

In particular, (5) holds for the following $r$ choices of $\delta_{1}, \ldots, \delta_{r}: \delta_{i_{0}}=1, \ldots, \delta_{j}=0$ for $j \neq i_{0}, i_{0}$ running from 1 to $r$. Therefore, for $t$ in the intersection $U$ of the corresponding $r$ neighbourhoods $U^{\prime}$ we have

$$
\mathbf{d}_{1}\left(E_{\mid C_{t}}\right) \leq \sum_{i=1}^{r} \mathbf{d}_{1}\left(E_{\mid C_{i}}\right) .
$$

Since $\mathbf{d}_{\mathrm{rk} E}(E)=-\mathbf{d}_{1}\left(E^{\vee}\right)$, inequality (6) applied to $E^{\vee}$ instead of $E$ yields

$$
\mathbf{d}_{\mathrm{rk} E}\left(E_{\mid C_{t}}\right) \geq \sum_{i=1}^{r} \mathbf{d}_{\mathrm{rk} E}\left(E_{\mid C_{i}}\right)
$$

for $t \in U$. The desired inequality (3) follows from (6) and (17).

We now proceed to the auxiliary statements used above. Given a strict partition $\left(n_{1}, \ldots, n_{r}\right)$ of $n \in \mathbf{Z}_{>0}$, we define a polarized chain (of rational cruves) as a pair $\left(C, \mathcal{O}_{C}\left(n_{1}, \ldots, n_{r}\right)\right)$, where $C=C_{1} \cup \ldots \cup C_{r}$ is a chain of rational curves.

Lemma 3.2. For any strict partition $\left(n_{1}, \ldots, n_{r}\right)$ of $n \in \mathbf{Z}_{>0}$ and any polarized chain $\left(C, \mathcal{O}_{C}\left(n_{1}, \ldots, n_{r}\right)\right)$ there exists a linearly normal 1 embedding $i: C \hookrightarrow \mathbb{P}^{n}$ such that $\left.\mathcal{O}_{C}\left(n_{1}, \ldots, n_{r}\right)\right) \simeq i^{*} \mathcal{O}_{\mathbb{P}^{n}}(1)$.

Proof. We use induction on $r$. For $r=1$ the desired embedding $i: C \hookrightarrow \mathbb{P}^{n}$ is clearly given by the complete linear series $\left|\mathcal{O}_{\mathbb{P}^{1}}\left(n_{1}\right)\right|$. Assume now that the claim is true for $r-1$. If we decompose $C$ as $C=C^{\prime} \cup C_{r}$, where $C^{\prime}:=C_{1} \cup \ldots \cup C_{r-1}$, and set $n^{\prime}:=n-n_{r}$, then by the induction assumption there exists a linearly normal embedding $i^{\prime}: C^{\prime} \hookrightarrow \mathbb{P}^{n^{\prime}}$ such that $\left.\mathcal{O}_{C}\left(n_{1}, \ldots, n_{r-1}\right)\right)=i^{\prime *} \mathcal{O}_{\mathbb{P}^{\prime \prime}}(1)$. Next, consider the linearly normal embedding $i_{r}: C_{r} \hookrightarrow \mathbb{P}^{r}$ by the complete linear series $\left|\mathcal{O}_{\mathbb{P}^{1}}\left(n_{r}\right)\right|$ and embed the spaces $\mathbb{P}^{n^{\prime}}=\operatorname{Span}\left(i^{\prime}\left(C^{\prime}\right)\right)$ and $\mathbb{P}^{r}=$ $\operatorname{Span}\left(i_{r}\left(C_{r}\right)\right)$ into the projective space $\mathbb{P}^{n}$ in such a way that their intersection $\mathbb{P}^{n^{\prime}} \cap \mathbb{P}^{r}$ in $\mathbb{P}^{n}$ is a point. We may assume, after possible projective linear transformations of $\mathbb{P}^{n^{\prime}}$ and $\mathbb{P}^{r}$, that this point equals $i^{\prime}\left(C^{\prime}\right) \cap i_{r}\left(C_{r}\right)$. Thus we obtain an embedding $i: C \hookrightarrow \mathbb{P}^{n}$ such that $i_{\mid C^{\prime}}=i^{\prime}$, $i_{\mid C_{r}}=i_{r}$ and, by the construction, $\left.\mathcal{O}_{C}\left(n_{1}, \ldots, n_{r}\right)\right)=i^{*} \mathcal{O}_{\mathbb{P}^{n}}(1)$.

\footnotetext{
${ }^{1}$ Recall that $i$ is linearly normal if $\operatorname{Span}(i(C))=\mathbb{P}^{n}$.
} 
Next, we recall that a $k$-pointed chain (of rational curves) is a datum $\left(C, B_{1}, \ldots, B_{k}\right)$ consisting of: (i) a chain $C=C_{1} \cup \ldots \cup C_{r}$ of rational curves, (ii) a set of $k$ distinct points $B_{1}, \ldots, B_{k} \in C$ which for $r>1$ are also distinct from the points $A_{i}=C_{i} \cap C_{i+1}, i=1, \ldots, r-1$. For any $k$-pointed chain $\left(C, B_{1}, \ldots, B_{k}\right)$ we denote its isomorphism class by $\left[\left(C, B_{1}, \ldots, B_{k}\right)\right]$. The set $\overline{M_{0, k}}$ of isomorphism classes of $k$-pointed chains is the well known moduli space of $k$-pointed chains (of rational curves).

Denote by Hilb ${ }^{n+1} \mathbb{P}^{n}$ the Hilbert scheme of subschemes of $\mathbb{P}^{n}$ with Hilbert polynomial $n+1$. Fix $n+2$ points $B_{1}, \ldots, B_{n+2} \in \mathbb{P}^{n}$ in general position, i.e. such that no $n+1$ points lie in a hyperplane of $\mathbb{P}^{n}$. Consider the set $V\left(B_{1}, \ldots, B_{n+2}\right):=\left\{C \in \operatorname{Hilb}^{n+1} \mathbb{P}^{n} \mid\left(C, B_{1}, \ldots, B_{k}\right)\right.$ is a $k$-pointed chain in $\left.\mathbb{P}^{n}\right\}$ and the morphism

$$
\theta: V\left(B_{1}, \ldots, B_{n+2}\right) \rightarrow \overline{M_{0, n+2}}, C \mapsto\left[\left(C, B_{1}, \ldots, B_{n+2}\right)\right] .
$$

In addition, put $\Gamma\left(B_{1}, \ldots, B_{n+2}\right):=\left\{(x, C) \in \mathbb{P}^{n} \times V\left(B_{1}, \ldots, B_{n+2}\right) \mid x \in C\right\}$.

Now we invoke results of Kapranov [K] concerning $k$-pointed chains. Together with Lemma 3.2 these results yield the following proposition.

\section{Proposition 3.3.}

1) The morphism $\theta$ is an isomorphism, hence it induces an embedding $i_{\Gamma}: \Gamma\left(B_{1}, \ldots, B_{n+2}\right) \hookrightarrow$ $\mathbb{P}^{n} \times \overline{M_{0, n+2}}$.

2) For any strict partition $\left(n_{1}, \ldots, n_{r}\right)$ of $n \in \mathbf{Z}_{>0}$ and any polarized chain $\left(C_{0}, \mathcal{O}_{C_{0}}\left(n_{1}, \ldots, n_{r}\right)\right)$, there exist points $B_{1}, \ldots, B_{n+2} \in C_{0}$ such that the point $c_{0}=\left[\left(C_{0}, B_{1}, \ldots, B_{n+2}\right)\right] \in \overline{M_{0, n+2}}$ satisfies the condition $\left.\mathcal{O}_{C_{0}}\left(n_{1}, \ldots, n_{r}\right) \simeq i_{\Gamma}^{*}\left(\mathcal{O}_{\mathbb{P}^{n}}(1) \otimes \mathcal{O}_{\overline{M_{0, n+2}}}\right)_{\mid C_{0} \times\left\{c_{0}\right\}}\right)$.

3) The family of curves $\pi_{\Gamma}: \Gamma\left(B_{1}, \ldots, B_{n+2}\right) \stackrel{i_{\Gamma}}{\hookrightarrow} \mathbb{P}^{n} \times \overline{M_{0, n+2}} \stackrel{p r_{2}}{\longrightarrow} \overline{M_{0, n+2}}$ is a semiuniversal deformation of the curve $C_{0}$, i.e., for any flat family $\pi: X \rightarrow B$ of chains of rational curves such that $C_{0}=\pi^{-1}(0)$ for some point $0 \in B$, there exists a neighbourhood $U \ni 0$ in $B$ and a morphism $f: U \rightarrow \overline{M_{0, n+2}}$ with $f(0)=c_{0}$ and $\pi^{-1}(U)=\Gamma\left(B_{1}, \ldots, B_{n+2}\right) \times \frac{}{M_{0, n+2}} U$.

4) The line bundle $\mathcal{L}:=\Phi^{*} i_{\Gamma}^{*}\left(\mathcal{O}_{\mathbb{P}^{n}}(1) \otimes \mathcal{O}_{\overline{M_{0, n+2}}}\right)$, where $\Phi: \pi^{-1}(U) \rightarrow \Gamma\left(B_{1}, \ldots, B_{n+2}\right)$ is the induced morphism, satisfies the property $\mathcal{L}_{\mid C_{0}} \simeq \mathcal{O}_{C_{0}}\left(n_{1}, \ldots, n_{r}\right)$.

Corollary 3.4. Let $\pi: X \rightarrow B$ be a flat family of chains of rational curves. Let $0 \in B$ be a fixed point. For any line bundle $L_{0}$ on the fiber $C_{0}=\pi^{-1}(0)$ there exists a neighbourhood $U \ni 0$ in $B$ and a line bundle $\mathcal{L}$ on $\pi^{-1}(U)$ such that $\mathcal{L}_{\mid C_{0}} \simeq L_{0}$.

Proof. If $L_{0}$ is ample, its restrictions to each irreducible component of the fiber $C_{0}$ define a strict partition $\left(n_{1}, \ldots, n_{r}\right)$, and our statement is an immediate consequence of Proposition 3.3, 2). Since any line bundle $L_{0}$ on $C_{0}$ can be represented as $L_{0}^{\prime} \otimes L_{0}^{\prime \prime \vee}$ for some ample bundles $L_{0}^{\prime}, L_{0}^{\prime \prime}$, the Corollary follows.

Lemma 3.5. Let $C=C_{1} \cup \ldots \cup C_{r}$, be a chain of rational curves and let $E$ be a vector bundle on $C$. Then, for any $\delta_{1}, \ldots, \delta_{r} \in \mathbf{Z}_{\geq 0}$ with $\sum_{i=1}^{r} \delta_{i}>0$, one has

$$
h^{0}\left(E\left(-\mathbf{d}_{1}\left(E_{\mid C_{1}}\right)-\delta_{1}, \ldots,-\mathbf{d}_{1}\left(E_{\mid C_{r}}\right)-\delta_{r}\right)\right)=0 .
$$

Proof. We use induction on $r$. For $r=1$ the statement is clear from the definition of $\mathbf{d}_{1}(E)$. For the step of induction we just consider the case $r=2$, since for arbitrary $r$ the argument goes through without changes. Let $r=2$ and $\delta_{1}>0$. Then clearly $h^{0}\left(E\left(-\mathbf{d}_{1}\left(E_{\mid C_{1}}\right)-\delta_{1}\right)\right)=0$. Hence the natural exact triple

$$
\begin{gathered}
0 \rightarrow\left(E_{\mid C_{2}}\right)\left(-\mathbf{d}_{1}\left(E_{\mid C_{2}}\right)-\delta_{2}-1\right) \rightarrow E\left(-\mathbf{d}_{1}\left(E_{\mid C_{1}}\right)-\delta_{1},-\mathbf{d}_{1}\left(E_{\mid C_{2}}\right)-\delta_{2}\right) \rightarrow \\
\rightarrow\left(E_{\mid C_{1}}\right)\left(-\mathbf{d}_{1}\left(E_{\mid C_{1}}\right)-\delta_{1}\right) \rightarrow 0
\end{gathered}
$$

implies the equality $h^{0}\left(E\left(-\mathbf{d}_{1}\left(E_{\mid C_{1}}\right)-\delta_{1},-\mathbf{d}_{1}\left(E_{\mid C_{2}}\right)-\delta_{2}\right)\right)=0$. 
4. Construction of special rational curves in $G(r, V)$

Let $E$ be a rank $k$ vector bundle on $G=G(r, V)$. Denote

$$
\mathbf{D}(E):=\max \left\{\mathbf{D}\left(E_{\mid l}\right) \mid l \text { is a line in } G\right\} .
$$

Our aim in this section is to prove that, for any point $y_{0} \in G$, the inequality $\mathbf{D}\left(E_{\mid C}\right) \leq 2 r \mathbf{D}(E)$ holds on a dense open subset of a suitably defined subscheme of the Hilbert scheme $H_{2 r}\left(y_{0}\right)$ of rational curves $C$ of degree $2 r$ on $G$ passing through the point $y_{0}$.

We start with the following construction. Under the assumption that $\operatorname{dim} V \geq 3 r$, let $V^{\prime}, V^{\prime \prime}, V^{\prime \prime \prime}$ be three $r$-dimensional subspaces of $V$ such that

$$
V^{\prime} \cap V^{\prime \prime}=V^{\prime} \cap V^{\prime \prime \prime}=V^{\prime \prime} \cap V^{\prime \prime \prime}=\{0\}, \quad V^{\prime \prime \prime} \subset V^{\prime} \oplus V^{\prime \prime} .
$$

In addition, fix $r$ linearly independent one-dimensional subspaces $V_{i}^{\prime}, i=1, \ldots, r$, in $V^{\prime}$. This datum defines linearly independent one-dimensional subspaces $V_{i}^{\prime \prime}:=V^{\prime \prime} \cap\left(V^{\prime \prime \prime} \oplus V_{i}^{\prime}\right)$ in $V^{\prime \prime}$, as well as linearly independent one- dimensional subspaces $V_{i}^{\prime \prime \prime}:=V^{\prime \prime \prime} \cap\left(V_{i}^{\prime} \oplus V_{i}^{\prime \prime}\right)$. We obtain $r$ projective lines $\mathbb{P}_{i}^{1}:=P\left(V_{i}^{\prime} \oplus V_{i}^{\prime \prime}\right)$, with points $V_{i}^{\prime}, V_{i}^{\prime \prime}, V_{i}^{\prime \prime \prime}, i=1, \ldots, r$, on them. On each of the lines $\mathbb{P}_{i}^{1}$ there is an affine coordinate $t_{i}$ uniquely determined by the condition

$$
V_{i}^{\prime}=\left\{t_{i}=0\right\}, \quad V_{i}^{\prime \prime}=\left\{t_{i}=\infty\right\}, \quad V_{i}^{\prime \prime \prime}=\left\{t_{i}=1\right\}, \quad i=1, \ldots, r .
$$

Let $V_{t i} \in \mathbb{P}_{i}^{1}$ be the point with affine coordinate $t$. By construction, the points $V_{t 1}, \ldots, V_{t r}$, considered as one-dimensional subspaces of $V$, are linearly independent in $V$ and their span $V_{t}^{r}:=V_{t 1} \oplus \ldots \oplus V_{t r}$ is an $r$-dimensional subspace of $V$. Thus we have an embedding

$$
\varphi_{1}: \mathbb{P}^{1} \hookrightarrow G, t \mapsto V_{t}^{r}
$$

such that $\varphi_{1}(0)=V^{\prime}, \varphi_{1}(\infty)=V^{\prime \prime}, \varphi_{1}(1)=V^{\prime \prime \prime}, \quad \varphi_{1}^{*} \mathcal{O}_{G}(1)=\mathcal{O}_{\mathbb{P}^{1}}(r)$. Note that the degree $r$ curve $C_{1}^{r}=\operatorname{im} \varphi_{1}$ depends only on the choice of the triple of $r$-dimensional spaces $V^{\prime}, V^{\prime \prime}, V^{\prime \prime \prime}$. We call the curve $C_{1}^{r}$ the Segre curve associated to $V^{\prime}, V^{\prime \prime}, V^{\prime \prime \prime}$. Moreover, the subspaces $V^{\prime}, V^{\prime \prime}, V^{\prime \prime \prime}$ define an embedding

$$
s=s\left(V^{\prime}, V^{\prime \prime}, V^{\prime \prime \prime}\right): \mathbb{P}^{1} \times \mathbb{P}^{r-1} \stackrel{s_{1,1}}{\hookrightarrow} \mathbb{P}^{2 r-1} \stackrel{j}{\hookrightarrow} P(V),
$$

where $s_{1,1}$ is the Segre embedding and $j$ is an embedding with $\operatorname{im} j=P\left(V^{\prime} \oplus V^{\prime \prime}\right)$. By construction, $\varphi_{1}(t)=s\left(V^{\prime}, V^{\prime \prime}, V^{\prime \prime \prime}\right)\left(\{t\} \times \mathbb{P}^{r-1}\right)$. We call $s\left(V^{\prime}, V^{\prime \prime}, V^{\prime \prime \prime}\right)$ the extended Segre embedding associated to $V^{\prime}, V^{\prime \prime}, V^{\prime \prime \prime}$.

More generally, for any $\left(t_{2}, \ldots, t_{r}\right) \in\left(\mathbf{k}^{*}\right)^{r-1}$ the triple of spaces $V^{\prime}, V^{\prime \prime}, V^{\left(t_{2}, \ldots, t_{r}\right)}$, where

$$
V^{\left(t_{2}, \ldots, t_{r}\right)}:=V_{11} \oplus V_{t_{2} 2} \oplus \ldots \oplus V_{t_{r} r}
$$

satisfies condition (8) with $V^{\prime \prime \prime}$ replaced by $V^{\left(t_{2}, \ldots, t_{r}\right)}$ and hence yields a Segre curve

$$
C_{\left(t_{2}, \ldots, t_{r}\right)}^{r}:=\operatorname{im} s\left(V^{\prime}, V^{\prime \prime}, V^{\left(t_{2}, \ldots, t_{r}\right)}\right)
$$

in $G$. In particular, $C_{(1, \ldots, 1)}^{r}$ coincides with the Segre curve $C_{1}^{r}=\operatorname{im} \varphi_{1}$. In addition,

$$
y_{0}:=\left\{V^{\prime}\right\} \in C_{\left(t_{2}, \ldots, t_{r}\right)}^{r} .
$$

Let $l_{1}$ be the line in $G$ determined by the flag $\left(V_{2}^{\prime} \oplus . . \oplus V_{r}^{\prime} \subset V_{1}^{\prime \prime} \oplus V_{1}^{\prime} \oplus \ldots \oplus V_{r}^{\prime}\right)$ and let $l_{i}$, for $i=2, \ldots, r$, be the line in $G$ determined by the flag $\left(V_{1}^{\prime \prime} \oplus \ldots \oplus V_{i-1}^{\prime \prime} \oplus V_{i+1}^{\prime} \oplus \ldots \oplus V_{r}^{\prime} \subset\right.$ $\left.V_{1}^{\prime \prime} \oplus \ldots \oplus V_{i}^{\prime \prime} \oplus V_{i}^{\prime} \oplus \ldots \oplus V_{r}^{\prime}\right)$. These lines constitute a chain of rational curves

$$
C_{0}^{r}=l_{1} \cup \ldots \cup l_{r}
$$

in $G$. Moreover, setting $\left(t_{2}, t_{3}, \ldots, t_{r}\right)=\left(t, t^{2}, \ldots, t^{r-1}\right)$, one easily proves the following lemma. 
Lemma 4.1. Let $G=G(r, V)$, with $\operatorname{dim} V \geq 3 r$. Consider the surface $\mathcal{S}^{\times}:=\{(x, t) \in$ $\left.G \times \mathbf{k}^{\times} \mid x \in C_{t}^{r}:=C_{\left(t, t^{2} \ldots, t^{r-1}\right)}^{r}\right\}$ with projection $\pi^{\times}: \mathcal{S}^{\times} \rightarrow \mathbf{k}^{\times},(x, t) \mapsto t$. Then the following statements hold.

(i) The fibers $C_{t}^{r}=\left(\pi^{\times}\right)^{-1}(t)$ are rational curves passing through the point $y_{0} \in G$.

(ii) Let $\mathbf{k}^{\times} \hookrightarrow \mathbf{k}=\mathbb{A}^{1}$ be the standard inclusion and let $\mathcal{S}$ be the closure of $\mathcal{S}^{\times}$in $G \times \mathbf{k}$. The extended projection $\pi: \mathcal{S} \rightarrow \mathbb{A}^{1}$ is a flat morphism and the natural morphism $\nu: \mathcal{S} \rightarrow G$, $(x, t) \mapsto x$ is birational and gives an isomorphism $\nu: \pi^{-1}(0) \stackrel{\sim}{\rightarrow} C_{0}^{r}$.

We note next that the condition $\operatorname{dim} V \geq 3 r$ in Lemma 4.1 can be removed. In fact, let $\operatorname{dim} V<3 r$. By our assumption (see Section 2), $r+2 \leq \operatorname{dim} V$. Fix a $3 r$-dimensional vector space $\tilde{V}$ and let $V^{\prime}, V^{\prime \prime}, V^{\prime \prime \prime}$ be three $r$-dimensional subspaces of $\tilde{V}$ satisfying the conditions (8). By performing the above constructions for this datum we obtain a surface $\tilde{\mathcal{S}}$ with projections $\mathbb{A}^{1} \stackrel{\tilde{\pi}}{\leftarrow} \tilde{\mathcal{S}} \stackrel{\tilde{\nu}}{\longrightarrow} \tilde{G}:=G(r, \tilde{V})$ as in Lemma 4.1. For $y \in \tilde{\mathcal{S}}$, let $V_{y}$ be the $r$-dimensional subspace of $\tilde{V}$ corresponding to the point $\tilde{\nu}(y)$ in $\tilde{G}$. Since $\operatorname{dim} \mathcal{S}=2$, it follows from the inequality $r+2 \leq \operatorname{dim} V$ that there exists a subspace $L$ of dimension $3 r-\operatorname{dim} V$ in $\tilde{V}$ such that

$$
L \cap\left(\underset{y \in \tilde{\mathcal{S}}}{\cup} V_{y}\right)=\{0\} .
$$

Fix an isomorphism $h: \tilde{V} / L \stackrel{\sim}{\rightarrow} V$ and consider the rational morphism $\tilde{f}: \tilde{G} \rightarrow G$, $\left.V^{r} \mapsto h\left(\left(V^{r}+L\right) \bmod L\right)\right)$. Then (10) implies that the morphism $f: \tilde{\mathcal{S}} \rightarrow G \times \mathbb{A}^{1},(x, t) \mapsto$ $(\tilde{f}(x), t)$ is an embedding, i.e. that there exists an isomorphism $g: \mathcal{S}:=f(\tilde{\mathcal{S}}) \stackrel{\sim}{\rightarrow} \tilde{S S}$ such that $f \circ g=\operatorname{id}_{\mathcal{S}}$. Hence the surface $\mathcal{S}$ with its projections $\pi:=\tilde{\pi} \circ g: \mathcal{S} \rightarrow \mathbb{A}^{1}$ and $\nu:=f \circ \tilde{\nu} \circ g: \mathcal{S} \rightarrow G$ satisfies the assertion of Lemma 4.1.

Theorem 3.1, Lemma 4.1 and this latest argument directly imply the following corollary.

Corollary 4.2. Let $E$ be a rank $k$ vector bundle on the Grassmannian $G$. There exists an open subset $U(E)$ of $\mathbf{k}^{*}$ such that, in the notation of Lemma 4.1, the inequality

$$
\mathbf{D}\left(E_{\mid C_{t}^{r}}\right) \leq r \mathbf{D}(E)
$$

holds for any $t \in U(E)$.

Fix $t \in U(E)$. According to (9), the extended Segre embedding $s_{t}:=s\left(V^{\prime}, V^{\prime \prime}, V^{\left(t, t^{2}, \ldots, t^{r-1}\right)}\right)$ : $\mathbb{P}^{1} \times \mathbb{P}^{r-1} \hookrightarrow P(V)$ induces an embedding $\psi_{t}: \mathbb{P}^{1} \rightarrow G, u \mapsto s_{t}\left(\{u\} \times \mathbb{P}^{r-1}\right)$, such that

$$
\operatorname{im} \psi_{t}=\pi^{-1}(t)=C_{t}^{r}
$$

is the Segre curve from Lemma 4.1. We will now construct another Segre curve $C^{\prime r}$ in $G$ such that $C_{t}^{r} \cup{C^{\prime}}_{t}^{\prime r}$ is a chain of rational curves (see (17) below).

For this, assume again temporarily that $\operatorname{dim} V \geq 3 r$. Set $W^{\prime \prime}:=V^{\prime \prime}, W_{i}^{\prime \prime}:=V_{i}^{\prime \prime}, i=1, \ldots, r$, and choose two $r$-dimensional subspaces $W^{\prime}, W^{\prime \prime \prime}$ in $V$ satisfying the conditions similar to (8)

$$
W^{\prime} \cap W^{\prime \prime}=W^{\prime} \cap W^{\prime \prime \prime}=W^{\prime \prime} \cap W^{\prime \prime \prime}=\{0\}, \quad W^{\prime \prime \prime} \subset W^{\prime} \oplus W^{\prime \prime},
$$

and the condition

$$
W^{\prime} \cap\left(V^{\prime} \oplus V^{\prime \prime}\right)=\{0\} .
$$

We repeat the above construction for the datum $\left(W^{\prime}, W^{\prime \prime}, W^{\prime \prime \prime}\right)$ instead of $\left(V^{\prime}, V^{\prime \prime}, V^{\prime \prime \prime}\right)$. First, there are uniquely defined linearly independent one-dimensional subspaces $W_{i}^{\prime}:=W^{\prime} \cap\left(W^{\prime \prime \prime} \oplus\right.$ $\left.W_{i}^{\prime \prime}\right)$ in $W^{\prime}$, as well as linearly independent one- dimensional subspaces $W_{i}^{\prime \prime \prime}:=W^{\prime \prime \prime} \cap\left(W^{\prime} \oplus W_{i}^{\prime \prime}\right)$ in $W^{\prime \prime \prime}$. Furthermore, for $i=1, \ldots, r$ there is a uniquely defined affine coordinate $t_{i}$ on the projective line $P\left(W_{i}^{\prime} \oplus W_{i}^{\prime \prime}\right)$ such that $W_{i}^{\prime}=\{t=0\}, \quad W_{i}^{\prime \prime}=\{t=\infty\}, \quad W_{i}^{\prime \prime \prime}=\{t=1\}$. Denote by $W_{t i}$ the point on $P\left(W_{i}^{\prime} \oplus W_{i}^{\prime \prime}\right)$ with coordinate $t_{i}$. Finally, set $W^{\left(t_{2}, \ldots, t_{r}\right)}:=W_{11} \oplus W_{t_{2} 2} \oplus$ $\ldots \oplus W_{t_{r} r},\left(t_{2}, \ldots, t_{r}\right) \in\left(\mathbf{k}^{*}\right)^{r-1}$. 
Next, for any $t \in \mathbf{k}^{*}$ consider the extended Segre embedding $s_{t}^{\prime}:=s\left(W^{\prime}, W^{\prime \prime}, W^{\left(t, t^{2}, \ldots, t^{r-1}\right)}\right)$ : $\mathbb{P}^{1^{\prime}} \times \mathbb{P}^{r-1} \hookrightarrow P(V)$, where $\mathbb{P}^{1^{\prime}}$ is a copy of $\mathbb{P}^{1}$. This yields a map $\psi_{t}^{\prime}: \mathbb{P}^{1^{\prime}} \rightarrow G, u \mapsto$ $s_{t}^{\prime}\left(\{u\} \times \mathbb{P}^{r-1}\right)$, such that

$$
C^{\prime r}=\operatorname{im} \psi_{t}^{\prime}
$$

is a degree- $r$ Segre curve and, as in Corollary 4.2, there exists an open subset $U^{\prime}(E)$ of $\mathbf{k}^{*}$ such that

$$
\mathbf{D}\left(E_{\mid C_{t}^{\prime r}}\right) \leq r \mathbf{D}(E), \quad t \in U^{\prime}(E) .
$$

Moreover, for $t \in U_{0}(E):=U(E) \cap U^{\prime}(E)$ we have $C_{t}^{r} \cap C^{\prime r}=\left\{V^{\prime \prime}\right\}$, i.e.

$$
C_{t}^{r} \cup C_{t}^{\prime r}, \quad t \in U_{0}(E),
$$

is a chain of rational curves in $G$ passing through the point $y_{0}=\left\{V^{\prime}\right\}$.

Note that the assumption $\operatorname{dim} V \geq 3 r$ can again be removed. Indeed, consider a $3 r$ dimensional space $\tilde{V}$ containing the $r$-dimensional subspaces $V^{\prime}, V^{\prime \prime}=W^{\prime \prime}, V^{\prime \prime \prime}, W^{\prime}, W^{\prime \prime \prime}$ satisfying (8), (13) and (14). Set $\tilde{G}:=G(r, \tilde{V})$ and let $\tilde{\mathcal{S}}^{\prime}$ be a surface in $\tilde{G} \times \mathbb{A}^{1}$ defined by $W^{\prime}, W^{\prime \prime}, W^{\prime \prime \prime}$ in the same way as $\tilde{\mathcal{S}}$ was defined by $V^{\prime}, V^{\prime \prime}, V^{\prime \prime \prime}$. Consider the subspace $L \subset \tilde{V}$ of dimension $3 r-\operatorname{dim} V$ satisfying the following condition similar to (10):

$$
L \cap\left(\underset{y \in \tilde{\mathcal{S}} \cup \tilde{\mathcal{S}}^{\prime}}{\bigcup} V_{y}\right)=\{0\} .
$$

Then the argument following Lemma 4.1 goes through without change, in particular respective morphisms $f^{\prime}: \tilde{\mathcal{S}} \cup \tilde{\mathcal{S}}^{\prime} \hookrightarrow G \times \mathbb{A}^{1}, \pi^{\prime}: \mathcal{S} \cup \mathcal{S}^{\prime}:=\operatorname{im} f \rightarrow \mathbb{A}^{1}$ and $\nu^{\prime}: \mathcal{S} \cup \mathcal{S}^{\prime} \rightarrow G$ are defined and the chain (17) is the image in $G$ of the chain $\left(\pi^{\prime}\right)^{-1}(0)$ under $\nu^{\prime}$.

As a next step we construct rational curves $C^{2 r}$ of degree $2 r$ in $G$ by deforming the chain (17).

Proposition 4.3. Let $E$ be a rank $k$ vector bundle on $G$ and $y_{0} \in G$ be an arbitrary point. There exists a rational degree $2 r$ curve $C$ in $G$ passing through the point $y_{0}$ such that:

(i) $\mathbf{D}\left(E_{\mid C}\right) \leq 2 r \mathbf{D}(E)$,

(ii) $Q_{\mid C} \simeq\left(\mathcal{O}_{C}(2)\right)^{r}$, where $Q$ is the antitautological bundle, i.e. the bundle dual to the tautological bundle on $G$.

Proof. Fix $t \in U_{0}(E)$. Assume first that $\operatorname{dim} V \geq 3 r$. Since $V^{\prime \prime}=W^{\prime \prime}$, the embeddings $s_{t}$ and $s_{t}^{\prime}$ define an embedding $Y \times \mathbb{P}^{r-1} \rightarrow P(V)$ where $Y=\mathbb{P}^{1} \cup \mathbb{P}^{1^{\prime}}$ as a reducible conic with singular point $w_{0}=\mathbb{P}^{1} \cap \mathbb{P}^{1^{\prime}}$ and a marked point $z_{0} \in \mathbb{P}^{1} \subset Y$ such that $\tilde{s}_{t}\left(\left\{w_{0}\right\} \times \mathbb{P}^{r-1}\right)=$ $P\left(V^{\prime \prime}\right), \quad \tilde{s}_{t}\left(\left\{z_{0}\right\} \times \mathbb{P}^{r-1}\right)=P\left(V^{\prime}\right)$. Note that, as a consequence of (14), there exists an embedding of $g: Y \hookrightarrow \mathbb{P}^{2}$ such that $\tilde{s}_{t}$ fits in the composition of maps

$$
\tilde{s}_{t}: Y \times \mathbb{P}^{r-1} \stackrel{g \times i d}{\hookrightarrow} \mathbb{P}^{2} \times \mathbb{P}^{r-1} \stackrel{s_{1,1}}{\hookrightarrow} \mathbb{P}^{3 r-1} \stackrel{j}{\hookrightarrow} P(V),
$$

where $s_{1,1}$ is the Segre embedding by the linear series $\left|\mathcal{O}_{\mathbb{P}^{2}}(1) \otimes \mathcal{O}_{\mathbb{P}^{r-1}}(1)\right|$ and $j$ is an embedding.

Now consider a pencil of conics $\left\{Y_{\tau}\right\}_{\tau \in \mathbb{P}^{1}}$ in $\mathbb{P}^{2}$ satisfying the conditions: (i) $Y_{0}=Y$, (ii) all conics of the pencil pass through the point $z_{0}$ and (iii) the generic conic in the pencil is smooth (i. e. a rational curve). Set $\mathcal{U}^{\prime}=\left\{\tau \in \mathbb{P}^{1} \backslash\{0\} \mid Y_{\tau}\right.$ is smooth $\}$ (this is a dense open subset of $\left.\mathbb{P}^{1}\right)$. In view of (18) there exists a dense open subset $\mathcal{U}^{*}$ of $\mathcal{U}^{\prime}$ such that, for any $\tau \in \mathcal{U}^{*}$ the composition $\tilde{s}_{t}: \mathbb{P}^{1} \times \mathbb{P}^{r-1} \simeq Y_{\tau} \times \mathbb{P}^{r-1} \hookrightarrow \mathbb{P}^{2} \times \mathbb{P}^{r-1} \stackrel{s_{1,1}}{\hookrightarrow} \mathbb{P}^{3 r-1} \stackrel{j}{\hookrightarrow} P(V)$, coincides with the embedding $f_{t, \tau}: \mathbb{P}^{1} \times \mathbb{P}^{r-1} \hookrightarrow P(V)$ by a subseries of the linear series $\left|\mathcal{O}_{\mathbb{P}^{1}}(2) \otimes \mathcal{O}_{\mathbb{P}^{r-1}}(1)\right|$. This implies that the induced map

$$
\varphi_{t, \tau}: \mathbb{P}^{1} \rightarrow G, u \mapsto f_{t, \tau}\left(\{u\} \times \mathbb{P}^{r-1}\right)
$$

satisfies the property

$$
\varphi_{t, \tau}^{*} Q \simeq\left(\mathcal{O}_{\mathbb{P}^{1}}(2)\right)^{r}, \quad \tau \in \mathcal{U}^{*}
$$


Put $\mathcal{U}:=\mathcal{U}^{*} \cup\{0\}$ and consider the total space $\Pi_{t}:=\left\{(x, \tau) \in \mathbb{P}^{2} \times \mathcal{U} \mid x \in Y_{\tau}\right\}$ of the above pencil of conics, together with the projections $\mathbb{P}^{2} \stackrel{\sigma}{\leftarrow} \Pi_{t} \stackrel{\rho}{\rightarrow} \mathcal{U}$. We obtain a morphism

$$
\varphi_{t}: \Pi_{t} \rightarrow G,(x, \tau) \mapsto s_{1,1}\left(\{x\} \times \mathbb{P}^{r-1}\right) .
$$

By construction, $\rho^{-1}(\tau)=Y_{\tau}$ and for $\tau \in \mathcal{U}^{*}$ the map $\varphi_{t \mid Y_{\tau}}$ coincides with $\varphi_{t, \tau}$ from (19). Moreover, by (12) and (15) we have an isomorphism

$$
\varphi_{t \mid Y_{0}}: Y_{0} \stackrel{\sim}{\rightarrow} C_{t, 0}^{2 r}:=C_{t}^{r} \cup C^{\prime r} .
$$

This means that $\varphi_{t \mid Y_{\tau}}$ is an embedding near $\tau=0$, i.e. the set $\mathcal{U}_{0}=\left\{\tau \in \mathcal{U} \mid Y_{\tau}\right.$ is smooth and $\varphi_{t \mid Y_{\tau}}$ is an embedding $\}$ is dense in $\mathcal{U}$. We thus obtain isomorphisms

$$
\varphi_{t, \tau}: \mathbb{P}^{1} \stackrel{\sim}{\rightarrow} C_{t, \tau}^{2 r}=\operatorname{im} \varphi_{t, \tau} \subset G, \quad \tau \in \mathcal{U}_{0} .
$$

The isomorphisms (21) and (22) show that $\left\{C_{t, \tau}^{2 r}\right\}_{\tau \in \mathcal{U}_{0} \cup\{0\}}$ is a flat family of curves in $G$ whose fiber at 0 is a chain of rational curves of the form $C_{t, 0}^{2 r}=C_{t}^{r} \cup C_{t}^{\prime r}$ and whose other fibers are rational curves $C_{t, \tau}^{2 r}$. Hence, applying Theorem 3.1 to $\varphi_{t, r}$ we obtain that $\mathcal{U}(E):=\{\tau \in$ $\left.\mathcal{U}_{0} \cup\{0\} \mid \mathbf{D}\left(E_{\mid C_{t, \tau}^{2 r}}\right) \leq \mathbf{D}\left(E_{\mid C_{t}^{r}}\right)+\mathbf{D}\left(E_{\mid C_{t}^{\prime r}}\right)\right\}$ is a dense open subset of $\mathcal{U}_{0} \cup\{0\}$. Combining this with (11) and (16), and using (20), we obtain the assertion of the Proposition for any curve $C:=C_{t, \tau}^{2 r},(t, \tau) \in U_{0}(E) \times \mathcal{U}_{0}(E)$.

Finally, it remains to remove the assumption $\operatorname{dim} V \geq 3 r$. Let $\operatorname{dim} V<3 r$. Take a space $\tilde{V}$ of dimension $3 r$ and choose its subspace $L$ of dimension $3 r-\operatorname{dim} V$ satisfying the condition

$$
P(L) \cap s_{1,1}\left(\mathbb{P}^{2} \times \mathbb{P}^{r-1}\right)=\emptyset,
$$

where $s_{1,1}$ is the Segre embedding defined in (18) and where the intersection is taken in the space $\mathbb{P}^{3 r-1}$ which is identified with $P(\tilde{V})$ in view of the condition (14). (Note that $L$ always exists as $\operatorname{dim} V \geq r+2$.) The rest of the argument goes through as in the remark preceeding the Proposition.

We are now ready to discuss Hilbert schemes. Recall that any rational curve of given degree $k$ in $G$ can be considered as a point in the Hilbert scheme Hilb ${ }^{k t+1} G$. Set

$$
\begin{gathered}
H_{k}:=\left\{C \in \operatorname{Hilb}^{k t+1} G \mid C \text { is a rational curve of degree } k \text { in } G\right\}, \\
R_{k}=\left\{\varphi: \mathbb{P}^{1} \rightarrow G \mid \varphi \text { is an embedding }\right\} .
\end{gathered}
$$

It is well known (see, e.g., [St, Theorem 2.1]) that $H_{k}$ is a smooth irreducible open subset of Hilb $^{k t+1} G$ and that the natural morphism

$$
g_{k}: R_{k} \rightarrow H_{k}, \varphi \mapsto \operatorname{im} \varphi
$$

is a principal $P G L(2)$-bundle. Next, consider the vector space $\operatorname{Hom}\left(V^{\vee}, \mathbf{k}^{r} \otimes H^{0}\left(\mathcal{O}_{\mathbb{P}^{1}}(2)\right)\right)$ and its dense open subset

$$
\begin{gathered}
W:=\left\{e \in \operatorname{Hom}\left(V^{\vee}, \mathbf{k}^{r} \otimes H^{0}\left(\mathcal{O}_{\mathbb{P}^{1}}(2)\right)\right) \mid\right. \text { the composition } \\
\left.\tilde{e}: V^{\vee} \otimes \mathcal{O}_{\mathbb{P}^{1}} \stackrel{e \otimes i d}{\longrightarrow} \mathbf{k}^{r} \otimes H^{0}\left(\mathcal{O}_{\mathbb{P}^{1}}(2)\right) \otimes \mathcal{O}_{\mathbb{P}^{1}} \stackrel{e v}{\longrightarrow} \mathbf{k}^{r} \otimes \mathcal{O}_{\mathbb{P}^{1}}(2) \text { is an epimorphism }\right\},
\end{gathered}
$$

where $e v$ is the evaluation map. Let $\gamma: V^{\vee} \otimes \mathcal{O}_{G} \rightarrow Q$ be the natural epimorphism. By the universality of the Grassmannian $G$ any element $e \in W$ defines a pair

$$
\left(\varphi_{e}: \mathbb{P}^{1} \rightarrow G, \quad \chi_{e}: \varphi_{e}^{*} Q \stackrel{\sim}{\rightarrow} \mathbf{k}^{r} \otimes \mathcal{O}_{\mathbb{P}^{1}}(2)\right)
$$

such that

$$
\chi_{e} \circ \varphi_{e}^{*} \gamma=\tilde{e}
$$

where $\tilde{e}$ is defined in (25). Conversely, the element $e$ is recovered by the pair $\left(\varphi_{e}, \chi_{e}\right)$ since clearly $e$ is obtained from $\tilde{e}$ by passing to sections:

$$
e=H^{0}(\tilde{e}) \text {. }
$$


Now put $k=2 r$ in (23) and consider the set

$$
H_{2 r}^{*}:=\left\{C \in H_{2 r} \mid \mathbf{D}_{Q}(C)=0 \text {, i.e. } Q_{\mid C} \simeq \mathbf{k}^{r} \otimes \mathcal{O}_{\mathbb{P}^{1}}(2)\right\} .
$$

By semicontinuity, $H_{2 r}^{*}$ is an open subset of $H_{2 r}$. Moreover, $H_{2 r}^{*}$ is nonempty (and hence dense in $H_{2 r}$ ) since it contains all curves $C_{t, \tau}^{2 r}$ from Proposition 4.3 .

Theorem 4.4. Fix a point $y_{0} \in G$ and put

$$
H_{2 r}^{*}\left(y_{0}\right):=\left\{C \in H_{2 r}^{*} \mid y_{0} \in C\right\} .
$$

For any vector bundle $E$ on $G, B\left(E, y_{0}\right):=\left\{C \in H_{2 r}^{*}\left(y_{0}\right) \mid \mathbf{D}\left(E_{C}\right) \leq 2 r \mathbf{D}(E)\right\}$ is a dense open subset of the irreducible variety $H_{2 r}^{*}\left(y_{0}\right)$.

Proof. Since $H_{2 r}^{*}$ is smooth and irreducible, and the group $P G L\left(V^{\vee}\right)$ acts transitively on $G$, it follows that $H_{2 r}^{*}\left(y_{0}\right)$ is an irreducible (and smooth) subvariety of $H_{2 r}^{*}$ which contains the curve $C=C_{t, \tau}^{2 r}$ from Proposition 4.3. Moreover, since the condition $\mathbf{D}\left(E_{C}\right) \leq 2 r \mathbf{D}(E)$ is open on $C \in H_{2 r}^{*}\left(y_{0}\right)$ by semicontinuity, Proposition 4.3 immediately implies the Theorem.

Now take an arbitrary curve $C \in H_{2 r}^{*}$ and pick an embedding $\varphi_{C}: \mathbb{P}^{1} \hookrightarrow G$ such that $\operatorname{im} \varphi_{C}=C$. In addition, pick an isomorphism $\chi_{C}: \varphi_{C}^{*} Q \stackrel{\sim}{\rightarrow} \mathbf{k}^{r} \otimes \mathcal{O}_{\mathbb{P}^{1}}(2)$. These data define an element $e=H^{0}\left(\chi_{C} \circ \varphi_{C}^{*} \gamma\right) \in W(\mathrm{cf}$. (27) and (28) $)$ such that $\varphi_{e}=\varphi_{C}$. Moreover, $e$ belongs to the subset

$$
W^{*}:=\left\{e \in W \mid \varphi_{e}: \mathbb{P}^{1} \rightarrow G \text { is an embedding }\right\}
$$

of $W$. This nonempty subset is clearly open in $W$, hence it is dense in $W$. Moreover, setting $R_{2 r}^{*}:=g_{2 r}^{-1}\left(H_{2 r}^{*}\right)$ (see (24)), we obtain a principal $G L(r)$-bundle $\theta_{2 r}: W^{*} \rightarrow R_{2 r}^{*}, e \mapsto \varphi_{e}$. Since (24) is a principal $P G L(2)$-bundle, the composition

$$
w_{2 r}:=g_{2 r} \circ \theta_{2 r}: W^{*} \rightarrow H_{2 r}^{*}
$$

is a smooth surjective morphism.

Fix a point $y_{0} \in G$ and consider the set

$$
W^{*}\left(E, y_{0}\right):=w_{2 r}^{-1}\left(B\left(E, y_{0}\right)\right)
$$

By (26), (30) and (29) any point $e \in W^{*}\left(E, y_{0}\right)$ defines an embedding $\varphi_{e}: \mathbb{P}^{1} \hookrightarrow G$ with $y_{0} \in \operatorname{im} \varphi_{e}$. Let $z:=\varphi_{e}^{-1}\left(y_{0}\right)$. We have an exact triple $0 \rightarrow \mathbf{k}^{r} \otimes \mathcal{O}_{\mathbb{P}^{1}}(1) \rightarrow \mathbf{k}^{r} \otimes \mathcal{O}_{\mathbb{P}^{1}}(2) \rightarrow$ $\mathbf{k}^{r} \otimes \mathbf{k}(z) \rightarrow 0$. By applying $\mathcal{H o m}\left(V^{\vee} \otimes \mathcal{O}_{\mathbb{P}^{1}},-\right)$ and passing to sections we obtain an exact triple

$$
0 \rightarrow \operatorname{Hom}\left(V^{\vee}, \mathbf{k}^{r} \otimes H^{0}\left(\mathcal{O}_{\mathbb{P}^{1}}(1)\right)\right) \rightarrow \operatorname{Hom}\left(V^{\vee}, \mathbf{k}^{r} \otimes H^{0}\left(\mathcal{O}_{\mathbb{P}^{1}}(2)\right)\right) \stackrel{\text { res }_{z}}{\longrightarrow} \operatorname{Hom}\left(V^{\vee}, \mathbf{k}^{r} \otimes \mathbf{k}(z)\right) \rightarrow 0 .
$$

By construction, the set

$$
W\left(E, y_{0}\right):=\operatorname{res}_{z}^{-1}\left(\operatorname{res}_{z}(e)\right) \simeq \operatorname{Hom}\left(V^{\vee}, \mathbf{k}^{r} \otimes H^{0}\left(\mathcal{O}_{\mathbb{P}^{1}}(1)\right)\right)
$$

depends only on $E$ and $y_{0}$ and contains $W^{*}\left(E, y_{0}\right)$ as a dense open subset. We thus obtain the following corollary.

Corollary 4.5. $W^{*}\left(E, y_{0}\right)=w_{2 r}^{-1}\left(B\left(E, y_{0}\right)\right)$ is a dense open subset of $W\left(E, y_{0}\right) \simeq$ $\operatorname{Hom}\left(V^{\vee}, \mathbf{k}^{r} \otimes H^{0}\left(\mathcal{O}_{\mathbb{P}^{1}}(1)\right)\right)$. 


\section{Construction of a special Vector bundle on $\mathbb{P}^{1} \times \mathbb{P}^{1}$}

A key ingredient in the proof of our main result, Theorem 7.1, is a specific morphism of $\mathbb{P}^{1} \times \mathbb{P}^{1}$ into $G=G(r, V)$. It defines a vector bundle of rank $r$ on $\mathbb{P}^{1} \times \mathbb{P}^{1}$ as the pullback of the antitautological bundle $Q$ on $G$. We construct this morphism in two steps. We first construct a suitable vector bundle on $\mathbb{P}^{1} \times \mathbb{P}^{1}$ and then prove that this bundle arises from an appropriate morphism of $\mathbb{P}^{1} \times \mathbb{P}^{1}$ to $G$.

We now proceed to the construction of a special vector bundle on $X:=\mathbb{P}^{1} \times \mathbb{P}^{1}$. Let $p r_{i}: X \rightarrow \mathbb{P}^{1}, i=1,2$, be the natural projections, and let 0 and $\infty$ be two fixed points on $\mathbb{P}^{1}$. Set $P:=p r_{1}^{-1}(0), \quad P^{\prime}:=p r_{1}^{-1}(\infty)$.

Fix a partition $\left(a_{1}, a_{2}, \ldots, a_{r}\right)$ of $d \in \mathbb{Z}_{\geq 0}$ such that $a_{1} \geq a_{2} \geq \ldots \geq a_{r}$, and consider a set $Z^{\prime}$ of $d-a_{1}$ distinct points on $\mathbb{P}^{1}$ of the form

$$
Z^{\prime}=\bigsqcup_{i=2}^{r} Z_{i}^{\prime}, \quad Z_{i}^{\prime}=\bigsqcup_{j=1}^{a_{i}} x_{i j}
$$

$\left(Z_{i}^{\prime} \neq \varnothing\right.$ for $\left.a_{i}=0\right)$. The set $Z^{\prime}$ determines the set

$$
Z:=P^{\prime} \cap p r_{2}^{-1}\left(Z^{\prime}\right)=\sqcup_{i=2}^{r} Z_{i}, \quad Z_{i}:=P^{\prime} \cap p r_{2}^{-1}\left(Z_{i}^{\prime}\right)=\stackrel{\sqcup}{j=1}_{a_{i}}\left(\infty, x_{i j}\right) .
$$

In what follows we think of $Z_{i}$ as reduced 0-dimensional subschemes of $X$.

Setting

$$
\mathcal{E}_{1}:=\mathcal{O}_{X}\left(0, a_{1}\right)
$$

we will recursively construct sheaves $\mathcal{E}_{k}$ for $2 \leq k \leq r$ via the exact triples

$$
0 \rightarrow \mathcal{E}_{k-1} \rightarrow \mathcal{E}_{k} \rightarrow \mathcal{I}_{Z_{k}, X}\left(0, a_{k}\right) \rightarrow 0, \quad 2 \leq k \leq r .
$$

Proposition 5.1. Assume that for $2 \leq k \leq r$ the sheaf $\mathcal{E}_{k-1}$ is locally free of rank $k-1$ and satisfies the conditions

$(38) h^{0}\left(\mathcal{E}_{k-1}(0,-1)\right)=a_{1}, \quad h^{0}\left(\mathcal{E}_{k-1}(1,-1)\right)=2 a_{1}+a_{2}+\ldots+a_{k-1}, \quad h^{0}\left(\mathcal{E}_{k-1}\right)=a_{1}+k-1$,

$h^{0}\left(\mathcal{E}_{k-1}(1,0)\right)=2 a_{1}+a_{2}+\ldots+a_{k-1}+2(k-1), \quad h^{0}\left(\mathcal{E}_{k-1}(2,0)\right)=3 a_{1}+2 a_{2}+\ldots+2 a_{k-1}+3(k-1)$,

$$
\begin{gathered}
h^{2}\left(\mathcal{E}_{k-1}\left(0,-a_{i}\right)\right)=0, \quad k \leq i \leq r, \\
h^{2}\left(\mathcal{E}_{k-1}(0,-1)\right)=0, \\
h^{1}\left(\mathcal{E}_{k-1}\right)=h^{1}\left(\mathcal{E}_{k-1}(0,-1)\right)=h^{1}\left(\mathcal{E}_{k-1}(1,0)\right)=0, \\
h^{1}\left(\mathcal{E}_{k-1}(1,-1)\right)=0,
\end{gathered}
$$

Then

(i) there is an epimorphism

$$
\operatorname{Ext}^{1}\left(\mathcal{I}_{Z_{k}, X}\left(0, a_{k}\right), \mathcal{E}_{k-1}\right) \stackrel{\beta}{\rightarrow} H^{0}\left(\mathcal{E} x t^{1}\left(\mathcal{I}_{Z_{k}, X}\left(0, a_{k}\right), \mathcal{E}_{k-1}\right)\right),
$$


and moreover

$$
H^{0}\left(\mathcal{E} x t^{1}\left(\mathcal{I}_{Z_{k}, X}\left(0, a_{k}\right), \mathcal{E}_{k-1}\right)\right) \simeq H^{0}\left(\mathcal{O}_{Z_{k}}^{k-1}\right) \simeq \underset{x \in Z_{k}}{\oplus} \mathbf{k}(x)^{k-1}
$$

(ii) there exists an element $\xi \in \operatorname{Ext}^{1}\left(\mathcal{I}_{Z_{k}, X}\left(0, a_{k}\right), \mathcal{E}_{k-1}\right)$ such that the sheaf $\mathcal{E}_{k}$ defined by the corresponding exact triple (33) is locally free of rank $k$ and satisfies the conditions (34)-(43) with $k$ substituted for $k-1$; we label the so modified conditions as (34')-(43').

Proof. (i) The existence of the epimorphism (44) follows from the standard exact sequence of local and global Ext's

$$
\begin{aligned}
0 \rightarrow H^{1}\left(\mathcal{H o m}\left(\mathcal{I}_{Z_{k}, X}\left(0, a_{k}\right), \mathcal{E}_{k-1}\right)\right) & \rightarrow \operatorname{Ext}^{1}\left(\mathcal{I}_{Z_{k}, X}\left(0, a_{k}\right), \mathcal{E}_{k-1}\right) \rightarrow H^{0}\left(\mathcal{E} x t^{1}\left(\mathcal{I}_{Z_{k}, X}\left(0, a_{k}\right), \mathcal{E}_{k-1}\right)\right) \rightarrow \\
\rightarrow & H^{2}\left(\mathcal{H o m}\left(\mathcal{I}_{Z_{k}, X}\left(0, a_{k}\right), \mathcal{E}_{k-1}\right)\right)
\end{aligned}
$$

and from (34) in view of the canonical isomorphism $\mathcal{H o m}\left(\mathcal{I}_{Z_{k}, X}\left(0, a_{k}\right), \mathcal{E}_{k-1}\right)=\mathcal{E}_{k-1}\left(0,-a_{k}\right)$. The isomorphisms in (45) are standard.

(ii) Pick an element $\xi \in \operatorname{Ext}^{1}\left(\mathcal{I}_{Z_{k}, X}\left(0, a_{k}\right), \mathcal{E}_{k-1}\right)$ and consider the extension (33) defined by $\xi$. Put $S_{x}:=p r_{2}^{-1}(x)$. If $x \notin Z_{2}^{\prime} \sqcup \ldots \sqcup Z_{k-1}^{\prime}$, then in view of (40) the restriction of (33) onto $S_{x}$ is $0 \rightarrow\left(\mathcal{O}_{\mathbb{P}^{1}}\right)^{k-1} \rightarrow \mathcal{E}_{k \mid S_{x}} \rightarrow \mathcal{O}_{\mathbb{P}^{1}} \rightarrow$ 0, i.e. $\mathcal{E}_{k \mid S_{x}} \simeq\left(\mathcal{O}_{\mathbb{P}^{1}}\right)^{k}$. This implies (40).

If $x \in Z_{2}^{\prime} \sqcup \ldots \sqcup Z_{k-1}^{\prime}$, then in view of (41) the restriction of (33) onto $S_{x}$ is $0 \rightarrow\left(\mathcal{O}_{\mathbb{P}^{1}}\right)^{k-3} \oplus$ $\mathcal{O}_{\mathbb{P}^{1}}(1) \oplus \mathcal{O}_{\mathbb{P}^{1}}(-1) \rightarrow \mathcal{E}_{k \mid S_{x}} \rightarrow \mathcal{O}_{\mathbb{P}^{1}} \rightarrow$ 0, i.e. $\mathcal{E}_{k \mid S_{x}} \simeq\left(\mathcal{O}_{\mathbb{P}^{1}}\right)^{k-2} \oplus \mathcal{O}_{\mathbb{P}^{1}}(1) \oplus \mathcal{O}_{\mathbb{P}^{1}}(-1)$, and we obtain (41) for $x \in Z_{2}^{\prime} \sqcup \ldots \sqcup Z_{k-1}^{\prime}$.

For $x \in Z_{k}^{\prime}$, one has $\mathcal{I}_{Z_{k}, X}\left(0, a_{k}\right)_{\mid S_{x}} \simeq \mathbf{k}(\bar{x}) \oplus \mathcal{O}_{\mathbb{P}^{1}}(-1)$, where $\bar{x}:=(\infty, x)$. Therefore (40) yields an exact sequence

$$
0 \rightarrow\left(\mathcal{O}_{\mathbb{P}^{1}}\right)^{k-1} \rightarrow \mathcal{E}_{k \mid S_{x}} \rightarrow \mathbf{k}(\bar{x}) \oplus \mathcal{O}_{\mathbb{P}^{1}}(-1) \rightarrow 0 .
$$

Here the extension (46) is given by an element

$$
\begin{gathered}
\xi_{x}^{1} \in \operatorname{Ext}^{1}\left(\mathcal{I}_{Z_{k}, X}\left(0, a_{k}\right)_{\mid S_{x}}, \mathcal{E}_{k-1 \mid S_{x}}\right) \simeq \operatorname{Ext}^{1}\left(\mathbf{k}(\bar{x}) \oplus \mathcal{O}_{\mathbb{P}^{1}}(-1),\left(\mathcal{O}_{\mathbb{P}^{1}}\right)^{k-1}\right) \simeq \\
\simeq H^{0}\left(\mathcal{E} x t^{1}\left(\mathbf{k}(\bar{x}) \oplus \mathcal{O}_{\mathbb{P}^{1}}(-1),\left(\mathcal{O}_{\mathbb{P}^{1}}\right)^{k-1}\right)\right) \simeq \mathbf{k}(\bar{x})^{k-1} .
\end{gathered}
$$

Note that a sufficient condition for (41) is that $\xi_{x}^{1} \neq 0$ for $x \in Z_{k}^{\prime}$. Note in addition that the restriction of (33) onto $S_{x}$ defines a natural restriction map

$$
\psi_{x}: \operatorname{Ext}^{1}\left(\mathcal{I}_{Z_{k}, X}\left(0, a_{k}\right), \mathcal{E}_{k-1}\right) \rightarrow \operatorname{Ext}^{1}\left(\mathcal{I}_{Z_{k}, X}\left(0, a_{k}\right)_{\mid S_{x}}, \mathcal{E}_{k-1 \mid S_{x}}\right)
$$

such that

$$
\xi_{x}^{1}=\psi_{x}(\xi) .
$$

The map $\psi_{x}$ together with (44) and (47) fits in the diagram

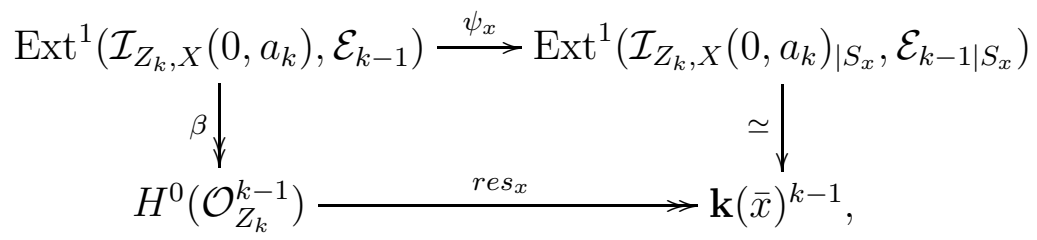

where $r e s_{x}$ is the restriction epimorphism defined by the inclusion $\bar{x} \hookrightarrow Z_{k}$.

Next, (33) and (42) give

$$
0 \rightarrow \mathcal{O}_{\mathbb{P}^{1}}\left(a_{1}\right) \oplus \ldots \oplus \mathcal{O}_{\mathbb{P}^{1}}\left(a_{k-1}\right) \rightarrow \mathcal{E}_{k \mid P} \rightarrow \mathcal{O}_{\mathbb{P}^{1}}\left(a_{k}\right) \rightarrow 0
$$

Since $a_{1} \geq a_{2} \geq \ldots \geq a_{k}$, this extension splits and yields (42). Furthermore, by (33) and (43) we have

$$
0 \rightarrow \mathcal{O}_{\mathbb{P}^{1}}\left(a_{1}+\ldots+a_{k-1}\right) \oplus\left(\mathcal{O}_{\mathbb{P}^{1}}\right)^{k-2} \rightarrow \mathcal{E}_{k \mid P^{\prime}} \rightarrow \mathcal{O}_{Z_{k}} \oplus \mathcal{O}_{\mathbb{P}^{1}} \rightarrow 0 .
$$

The extension (49) is given by an element

(50) $\xi^{\prime} \in \operatorname{Ext}^{1}\left(\mathcal{I}_{Z_{k}, X}\left(0, a_{k}\right)_{\mid P^{\prime}}, \mathcal{E}_{k-1 \mid P^{\prime}}\right) \simeq \operatorname{Ext}^{1}\left(\mathcal{O}_{Z_{k}} \oplus \mathcal{O}_{\mathbb{P}^{1}}, \mathcal{O}_{\mathbb{P}^{1}}\left(a_{1}+\ldots+a_{k-1}\right) \oplus\left(\mathcal{O}_{\mathbb{P}^{1}}\right)^{k-2}\right) \simeq$ 


$$
\simeq H^{0}\left(\mathcal{O}_{Z_{k}}^{k-1}\right)
$$

Since $a_{1}+\ldots+a_{k-1} \geq 0$, there is a distinguished injection

$$
\begin{aligned}
g_{k}=\bigoplus_{x \in Z_{k}} g_{k}(x): \bigoplus_{x \in Z_{k}} \mathbf{k}(x) \simeq \operatorname{Ext}^{1}\left(\mathcal{O}_{Z_{k}} \oplus \mathcal{O}_{\mathbb{P}^{1}}, \mathcal{O}_{\mathbb{P}^{1}}\left(a_{1}+\ldots+a_{k-1}\right)\right) \hookrightarrow \operatorname{Ext}^{1}\left(\mathcal{I}_{Z_{k}, X}\left(0, a_{k}\right)_{\mid P^{\prime}}, \mathcal{E}_{k-1 \mid P^{\prime}}\right) \\
\simeq \underset{x \in Z_{k}}{\bigoplus \mathbf{k}}(x)^{k-1} .
\end{aligned}
$$

Furthermore, in view of (44) and (50), we have a diagram of morphisms similar to (48)

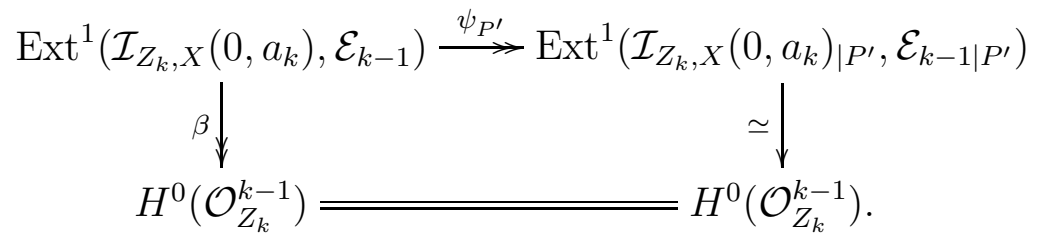

The diagrams (48) and (151) immediately imply that the element $\xi \in \operatorname{Ext}^{1}\left(\mathcal{I}_{Z_{k}, X}\left(0, a_{k}\right), \mathcal{E}_{k-1}\right)$ can be chosen so that:

1) $\left(r e s_{x} \circ \beta\right)(\xi) \neq 0$ for any $x \in Z_{k}$,

2) the element $\xi^{\prime}$ in (50) satisfies the condition $\xi^{\prime}=\psi_{P^{\prime}}(\xi) \in \operatorname{im} g_{k}$.

It follows from these conditions that (41) holds and the extension (49) implies (43).

To prove the remaining equalities (34)-(39) for the vector bundle $\mathcal{E}_{k}$ defined by $\xi$ as the extension (33), we consider the standard Koszul resolution

$$
0 \rightarrow \mathcal{O}_{X}(-1,0) \rightarrow \mathcal{O}_{X} \oplus \mathcal{O}_{X}\left(-1, a_{k}\right) \rightarrow \mathcal{I}_{Z_{k}, X}\left(0, a_{k}\right) \rightarrow 0
$$

Twisting (52) by $\mathcal{O}_{X}(a, b)$ for appropriate $a, b$ and keeping in mind that $a_{k} \geq 0$, we obtain

$$
\begin{gathered}
h^{2}\left(\mathcal{I}_{Z_{k}, X}\left(0, a_{k}-a_{i}\right)\right)=0, \quad k+1 \leq i \leq r, \\
h^{2}\left(\mathcal{I}_{Z_{k}, X}\left(0, a_{k}-1\right)\right)=0, \\
h^{1}\left(\mathcal{I}_{Z_{k}, X}\left(0, a_{k}\right)\right)=h^{1}\left(\mathcal{I}_{Z_{k}, X}\left(1, a_{k}\right)\right)=0, \\
h^{1}\left(\mathcal{I}_{Z_{k}, X}\left(1, a_{k}-1\right)\right)=0, \\
h^{0}\left(\mathcal{I}_{Z_{k}, X}\left(0, a_{k}-1\right)\right)=0, \quad h^{0}\left(\mathcal{I}_{Z_{k}, X}\left(1, a_{k}-1\right)\right)=a_{k}, \quad h^{0}\left(\mathcal{I}_{Z_{k}, X}\left(0, a_{k}\right)\right)=1, \\
h^{0}\left(\mathcal{I}_{Z_{k}, X}\left(1, a_{k}\right)\right)=a_{k}+2, \quad h^{0}\left(\mathcal{I}_{Z_{k}, X}\left(1, a_{k}\right)\right)=2 a_{k}+3 .
\end{gathered}
$$

Furthermore, twisting (33) by $\mathcal{O}_{X}(a, b)$ we quickly see that: (34) and (53) imply (34); (35) and (54) imply (35); (36) and (55) imply (36); (37) and (156) imply (37); (38) and (57) imply (38); (39) and (58) imply (39). The Proposition is proved.

As a corollary of Proposition 5.1 one obtains the following theorem.

Theorem 5.2. For any $r \in \mathbf{Z}_{>0}, r \geq 2$, there exists a rank $r$ vector bundle $\mathcal{F}$ on the surface $X=\mathbb{P}^{1} \times \mathbb{P}^{1}$ with the following properties:

$$
\begin{gathered}
\mathcal{F}_{\mid p r_{2}^{-1}(x)}=\left(\mathcal{O}_{\mathbb{P}^{1}}(2)\right)^{r}, \quad x \notin Z^{\prime}, \\
\mathcal{F}_{\mid p r_{2}^{-1}(x)}=\left(\mathcal{O}_{\mathbb{P}^{1}}(2)\right)^{r-2} \oplus \mathcal{O}_{\mathbb{P}^{1}}(3) \oplus \mathcal{O}_{\mathbb{P}^{1}}(1), \quad x \in Z^{\prime}, \\
\mathcal{F}_{\mid P}=\mathcal{O}_{\mathbb{P}^{1}}\left(a_{1}\right) \oplus \ldots \oplus \mathcal{O}_{\mathbb{P}^{1}}\left(a_{r}\right), \\
\mathcal{F}_{\mid P^{\prime}}=\mathcal{O}_{\mathbb{P}^{1}}(d) \oplus\left(\mathcal{O}_{\mathbb{P}^{1}}\right)^{r-1}, \\
h^{1}(\mathcal{F}(-1,-1))=h^{1}(\mathcal{F}(-2,0))=h^{1}(\mathcal{F}(-2,-1))=h^{1}(\mathcal{F}(-1,0))=0,
\end{gathered}
$$




$$
\begin{gathered}
h^{2}(\mathcal{F}(-2,-1))=0, \\
h^{0}(\mathcal{F}(-1,-1))=a_{1}+d, \quad h^{0}(\mathcal{F}(-1,0))=a_{1}+d+2 r, \quad h^{0}(\mathcal{F})=a_{1}+2 d+3 r .
\end{gathered}
$$

Proof. We define $\mathcal{F}$ as $\mathcal{E}_{r}(2,0)$. The equalities (59)-(65) follow directly from the equalities (32) and (34)-(43) for $k=r$ and from the observation that $a_{1}+\ldots+a_{r}=d$.

\section{Construction of a special morphism $f: \mathbb{P}^{1} \times \mathbb{P}^{1} \rightarrow G(r, V)$}

We are now ready to proceed with the construction of the desired morphism $f: X \rightarrow G$. Fix a line $l_{0}$ in $G$, a point $y_{0} \in l_{0}$ and a degree $d$ morphism $\psi^{\prime}: \mathbb{P}^{1} \rightarrow l_{0}$, and let

$$
\psi: \mathbb{P}^{1} \stackrel{\psi^{\prime}}{\rightarrow} l_{0} \hookrightarrow G
$$

be the composition. The restriction of the canonical epimorphism $\gamma: V^{\vee} \otimes \mathcal{O}_{G} \rightarrow Q$ to $l_{0}$ has the form $g: V^{\vee} \otimes \mathcal{O}_{\mathbb{P}^{1}} \rightarrow \mathcal{O}_{\mathbb{P}^{1}}(1) \oplus\left(\mathcal{O}_{\mathbb{P}^{1}}\right)^{r-1}$. Hence the epimorphism $\psi^{*} g$ has the form

$$
\psi^{*} g: V^{\vee} \otimes \mathcal{O}_{\mathbb{P}^{1}} \rightarrow \mathcal{O}_{\mathbb{P}^{1}}(d) \oplus\left(\mathcal{O}_{\mathbb{P}^{1}}\right)^{r-1} .
$$

Passing to sections we obtain an element

$$
g_{\psi} \in \operatorname{Hom}\left(V^{\vee}, H^{0}\left(\mathcal{O}_{\mathbb{P}^{1}}(d) \oplus\left(\mathcal{O}_{\mathbb{P}^{1}}\right)^{r-1}\right)\right) .
$$

Note that, similarly to (26), $\psi$ is determined by the element $g_{\psi}$.

Next, we put $k=d$ in (23) and we fix a curve $C_{0} \in H_{d}$ together with an isomorphism $\theta: \mathbb{P}^{1} \stackrel{\sim}{\rightarrow} C_{0}$. The composition $\varphi_{C_{0}}: \mathbb{P}^{1} \stackrel{\theta}{\rightarrow} C_{0} \hookrightarrow G$ defines an epimorphism $\varphi_{C_{0}}^{*} \gamma: V^{\vee} \otimes \mathcal{O}_{\mathbb{P}^{1}} \rightarrow$ $\varphi_{C_{0}}^{*} Q$. Moreover,

$$
\varphi_{C_{0}}^{*} Q \simeq \mathcal{O}_{\mathbb{P}^{1}}\left(a_{1}\right) \oplus \ldots \oplus \mathcal{O}_{\mathbb{P}^{1}}\left(a_{r}\right)
$$

for some partition $\left(a_{1}, \ldots, a_{r}\right)$ of $d$. (The nonnegativity of the integers $a_{i}$ follows from the surjectivity of $\varphi_{C_{0}}^{*} \gamma$.) Pick an isomorphism $\chi_{C_{0}}: \varphi_{C_{0}}^{*} Q \stackrel{\sim}{\rightarrow} \mathcal{O}_{\mathbb{P}^{1}}\left(a_{1}\right) \oplus \ldots \oplus \mathcal{O}_{\mathbb{P}^{1}}\left(a_{r}\right)$. The datum $\left(\varphi_{C_{0}}, \chi_{C_{0}}\right)$ defines an element

$$
e\left(C_{0}\right)=H^{0}\left(\chi_{C_{0}} \circ \varphi_{C_{0}}^{*} \gamma\right) \in W\left(C_{0}\right):=\operatorname{Hom}\left(V^{\vee}, H^{0}\left(\mathcal{O}_{\mathbb{P}^{1}}\left(a_{1}\right) \oplus \ldots \oplus \mathcal{O}_{\mathbb{P}^{1}}\left(a_{r}\right)\right)\right)
$$

(cf. (27) and (28)).

The set $W\left(C_{0}\right)^{e p i}:=\left\{e \in W\left(C_{0}\right) \mid\right.$ the composition $V^{\vee} \otimes \mathcal{O}_{\mathbb{P}^{1}} \stackrel{e \otimes i d}{\rightarrow} H^{0}\left(\mathcal{O}_{\mathbb{P}^{1}}\left(a_{1}\right) \oplus \ldots \oplus\right.$ $\left.\mathcal{O}_{\mathbb{P}^{1}}\left(a_{r}\right)\right) \otimes \mathcal{O}_{\mathbb{P}^{1}} \stackrel{e v}{\rightarrow} \mathcal{O}_{\mathbb{P}^{1}}\left(a_{1}\right) \oplus \ldots \oplus \mathcal{O}_{\mathbb{P}^{1}}\left(a_{r}\right)$ is an epimorphism $\}$ is a dense open subset of $W\left(C_{0}\right)$ containing $e\left(C_{0}\right)$, and (by the universality property of $G$ ) any element $e \in W\left(C_{0}\right)^{e p i}$ determines a morphism

$$
\varphi_{e}: \mathbb{P}^{1} \rightarrow G
$$

(cf. (26)) . For any vector bundle $E$ on $G$ we put

$$
B_{P}\left(E, C_{0}\right):=\left\{C \in H_{d} \mid \mathbf{D}\left(E_{\mid C}\right) \leq \mathbf{D}\left(E_{\mid C_{0}}\right)\right\} .
$$

By construction $C_{0} \in B_{P}\left(E, C_{0}\right)$, and moreover by semicontinuity, $B_{P}\left(E, C_{0}\right)$ is an open subset of $H_{d}$. Since $e\left(C_{0}\right) \in W\left(C_{0}\right)^{e p i}$ it follows that

$$
W_{P}\left(E, C_{0}\right):=\left\{e \in W\left(C_{0}\right)^{e p i} \mid \operatorname{im} \varphi_{e} \in B_{P}\left(E, C_{0}\right)\right\}
$$

is a dense open subset in $W\left(C_{0}\right)^{e p i}$, respectively, in $W\left(C_{0}\right)$, and we obtain a natural surjection $W_{P}\left(E, C_{0}\right) \rightarrow B_{P}\left(E, C_{0}\right), e \mapsto \operatorname{im}\left(\varphi_{e}\right)$.

We put also

$$
\pi:=\left(p r_{2 \mid P}\right)^{-1}: \mathbb{P}^{1} \stackrel{\sim}{\rightarrow} P, \quad \pi^{\prime}:=\left(p r_{2 \mid P^{\prime}}\right)^{-1}: \mathbb{P}^{1} \stackrel{\sim}{\rightarrow} P^{\prime}, \quad \rho:=\left(p r_{1 \mid S}\right)^{-1}: \mathbb{P}^{1} \stackrel{\sim}{\rightarrow} S .
$$


Theorem 6.1. Let $y_{0} \in l_{0}$ and $w \in \psi^{-1}\left(y_{0}\right)$ be fixed points. Then, for any vector bundle $E$ on $G$ there exists a morphism $f: X \rightarrow G$ such that:

(i) $f^{*} Q \simeq \mathcal{F}$, where $\mathcal{F}$ is defined in Theorem 5.2;

(ii) $f \circ \pi^{\prime}=\psi$;

(iii) $f \circ \pi: \mathbb{P}^{1} \hookrightarrow G$ is an embedding such that $(f \circ \pi)^{*} Q \simeq \mathcal{O}_{\mathbb{P}^{1}}\left(a_{1}\right) \oplus \ldots \oplus \mathcal{O}_{\mathbb{P}^{1}}\left(a_{r}\right)$, respectively, $f \circ \rho: \mathbb{P}^{1} \hookrightarrow G$ is an embedding such that $(f \circ \rho)^{*} Q \simeq\left(\mathcal{O}_{\mathbb{P}^{1}}(2)\right)^{r}$;

(iv) $\mathbf{D}\left(E_{\mid f(P)}\right) \leq \mathbf{D}\left(E_{\mid C_{0}}\right)$ and $\mathbf{D}\left(E_{\mid f(S)}\right) \leq 2 r \mathbf{D}(E)$.
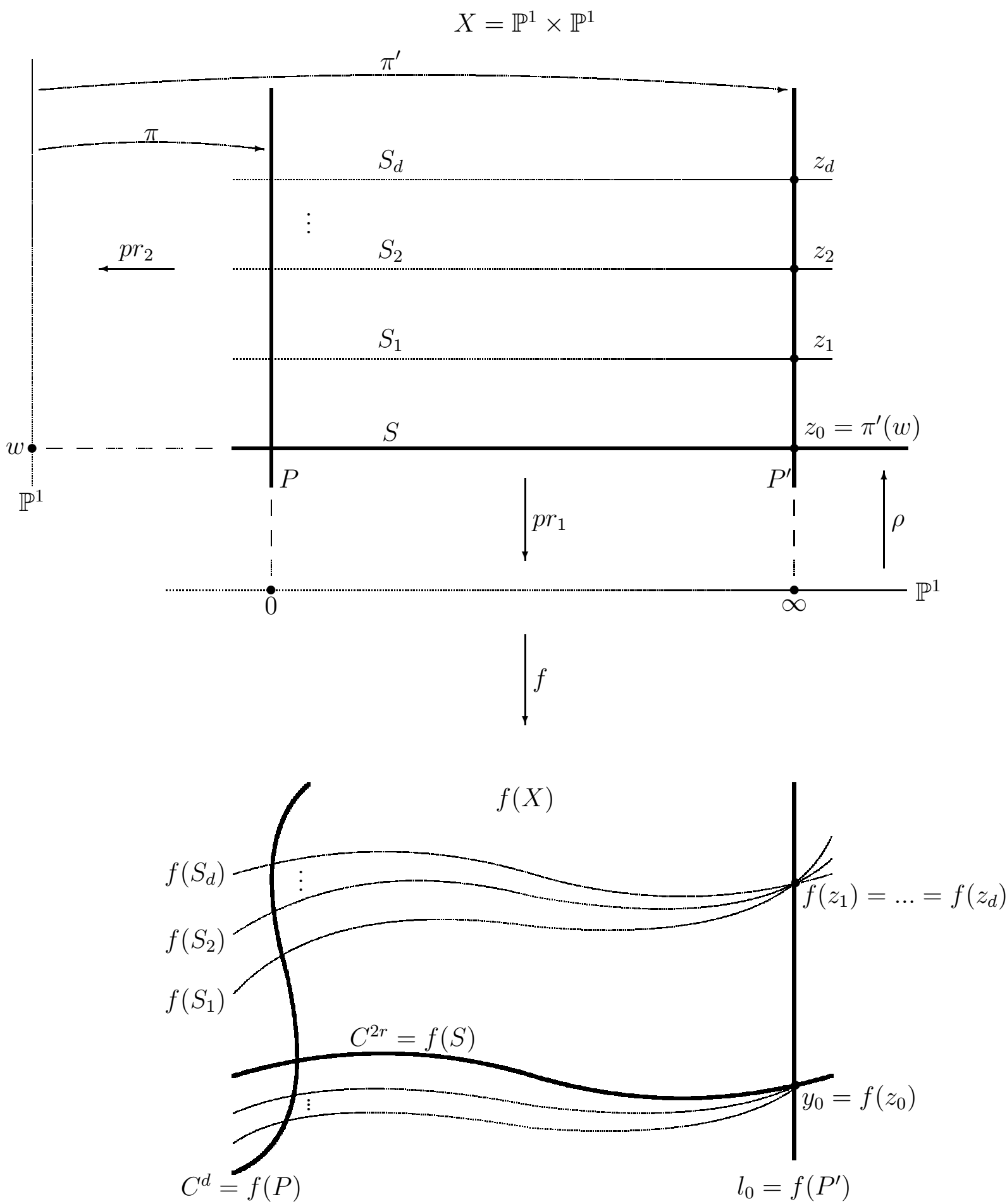

Proof. Recall (Theorem 5.2) that

$$
\mathcal{F}_{\mid P} \simeq \mathcal{O}_{\mathbb{P}^{1}}\left(a_{1}\right) \oplus \ldots \oplus \mathcal{O}_{\mathbb{P}^{1}}\left(a_{r}\right), \quad \mathcal{F}_{\mid P^{\prime}} \simeq \mathcal{O}_{\mathbb{P}^{1}}(d) \oplus\left(\mathcal{O}_{\mathbb{P}^{1}}\right)^{r-1}, \quad \mathcal{F}_{\mid S} \simeq\left(\mathcal{O}_{\mathbb{P}^{1}}(2)\right)^{r}
$$


Furthermore, let us introduce the following notation:

$$
\begin{gathered}
z_{0}:=P^{\prime} \cap S, \quad W_{0}:=\operatorname{Hom}\left(V^{\vee}, H^{0}(\mathcal{F})\right), \quad W_{-1}:=\operatorname{Hom}\left(V^{\vee}, H^{0}(\mathcal{F}(-1,0))\right), \\
W_{P}:=\operatorname{Hom}\left(V^{\vee}, H^{0}\left(\mathcal{F}_{\mid P}\right)\right), \quad W_{-1, P}:=\operatorname{Hom}\left(V^{\vee}, H^{0}\left(\mathcal{F}(-1,0)_{\mid P}\right)\right) \simeq W_{P}, \\
W_{0, P S}:=\operatorname{Hom}\left(V^{\vee}, H^{0}\left(\mathcal{F}_{\mid P \cup S}\right)\right), \quad W_{-1, P S}:=\operatorname{Hom}\left(V^{\vee}, H^{0}\left(\mathcal{F}(-1,0)_{\mid P \cup S}\right)\right), \\
W_{-1, S}:=\operatorname{Hom}\left(V^{\vee}, H^{0}\left(\mathcal{F}(-1,0)_{\mid S}\right)\right) \simeq \operatorname{Hom}\left(V^{\vee}, H^{0}\left(\left(\mathcal{O}_{\mathbb{P}^{1}}(1)\right)^{r}\right)\right), \\
W_{P^{\prime}}:=\operatorname{Hom}\left(V^{\vee}, H^{0}\left(\mathcal{F}_{\mid P^{\prime}}\right)\right) \simeq \operatorname{Hom}\left(V^{\vee}, H^{0}\left(\mathcal{O}_{\mathbb{P}^{1}}(d) \oplus\left(\mathcal{O}_{\mathbb{P}^{1}}\right)^{r-1}\right)\right) .
\end{gathered}
$$

(The right-hand isomorphisms in (771) and (72) follow from (69)).

By using (69), we see that the functor $\mathcal{H} m_{\mathcal{O}_{X}}\left(V^{\vee} \otimes \mathcal{O}_{X},-\right)$ applied to the exact sequence $0 \rightarrow \mathcal{F}(-1,0) \rightarrow \mathcal{F} \rightarrow \mathcal{F}_{\mid P^{\prime}} \rightarrow 0$ yields and exact sequence $0 \rightarrow \mathcal{H} m_{\mathcal{O}_{X}}\left(V^{\vee} \otimes \mathcal{O}_{X}, \mathcal{F}(-1,0)\right) \rightarrow$ $\mathcal{H o m}_{\mathcal{O}_{X}}\left(V^{\vee} \otimes \mathcal{O}_{X}, \mathcal{F}\right) \rightarrow \mathcal{H o m}_{\mathcal{O}_{P^{\prime}}}\left(V^{\vee} \otimes \mathcal{O}_{\mathbb{P}^{1}}, \mathcal{O}_{\mathbb{P}^{1}}(d) \oplus\left(\mathcal{O}_{\mathbb{P}^{1}}\right)^{r-1}\right) \rightarrow 0$. Passing to cohomology and using (63), we obtain the exact sequence

$$
0 \rightarrow W_{-1} \stackrel{i_{W}}{\rightarrow} W_{0} \stackrel{\text { res }_{P^{\prime}}}{\rightarrow} W_{P^{\prime}} \rightarrow 0 .
$$

Next, for any $s \in W_{-1}$ we consider the composition morphism $e_{s}: V^{\vee} \otimes \mathcal{O}_{X} \stackrel{s \otimes i d}{\longrightarrow} H^{0}(\mathcal{F}(-1,0)) \otimes$ $\mathcal{O}_{X} \stackrel{e v}{\longrightarrow} \mathcal{F}(-1,0)$, and, for any $z \in X \backslash P^{\prime}$, we consider the composition

$$
e_{s}(z): V^{\vee} \otimes \mathcal{O}_{X} \stackrel{e_{s}}{\rightarrow} \mathcal{F}(-1,0) \stackrel{\text { res }_{z}}{\rightarrow} \mathcal{F}(-1,0) \otimes \mathbf{k}(z) \simeq \mathbf{k}(z)^{r} .
$$

Passing to sections in the exact sequence $0 \rightarrow \mathcal{F}(-2,-1) \rightarrow \mathcal{F}(-2,0) \oplus \mathcal{F}(-1,-1) \rightarrow$ $\mathcal{F}(-1,0) \stackrel{\text { res } z}{\longrightarrow} \mathcal{F}(-1,0) \otimes \mathbf{k}(z) \rightarrow 0$ and using (63) and (64), we obtain an epimorphism $H^{0}(\mathcal{F}(-1,0)) \stackrel{\text { res }_{z}}{\rightarrow} H^{0}(\mathcal{F}(-1,0) \otimes \mathbf{k}(z)) \simeq \mathbf{k}^{r}$, and hence an induced epimorphism

$$
r(z): W_{-1} \rightarrow \operatorname{Hom}\left(V^{\vee}, H^{0}(\mathcal{F}(-1,0) \otimes \mathbf{k}(z))\right) \simeq \operatorname{Hom}\left(V^{\vee}, \mathbf{k}(z)^{r}\right)=: W_{z} .
$$

Put $Y(z):=\left\{s \in W_{-1} \mid e_{s}(z)\right.$ is not surjective $\}$ and $Y_{0}(z):=\left\{u \in W_{z} \mid u: V^{\vee} \rightarrow\right.$ $\mathbf{k}(z)^{r}$ is not surjective $\}$. By definition $Y(z)=r(z)^{-1}\left(Y_{0}(z)\right)$, and one easily checks that $\operatorname{codim}_{W_{z}} Y_{0}(z)=\operatorname{dim} V-r+1$. Therefore the surjectivity of $r(z)$ yields

$$
\operatorname{codim}_{W_{-1}} Y(z)=\operatorname{codim}_{W_{z}} Y_{0}(z)=\operatorname{dim} V-r+1 .
$$

If $Y:=\underset{z \in X \backslash P^{\prime}}{\cup} Y(z)$, (175) implies

$$
\operatorname{codim}_{W_{-1}} Y \geq \operatorname{dim} V-r-1>0 .
$$

(Note that $\operatorname{dim} V-r-1>0$ according to our assumption from Section 2.)

For each $z \in X \backslash P^{\prime}$ the exact sequence (73) and the map (74) fit in the diagram

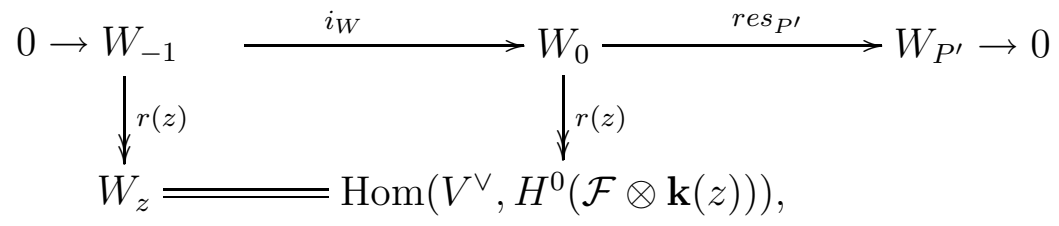

the right vertical map $r(z)$ being the natural restriction map. This diagram together with (72) and the inequality (76) shows that, for the element

$$
g_{\psi} \in \operatorname{Hom}\left(V^{\vee}, H^{0}\left(\mathcal{O}_{\mathbb{P}^{1}}(d) \oplus\left(\mathcal{O}_{\mathbb{P}^{1}}\right)^{r-1}\right)\right)=W_{P^{\prime}}
$$

given in (66) and for a generic element

$$
\varepsilon \in \operatorname{res}_{P^{\prime}}^{-1}\left(g_{\psi}\right) \simeq W_{-1},
$$

the composition $\varepsilon(z): V^{\vee} \otimes \mathcal{O}_{X} \stackrel{\varepsilon \otimes i d}{\longrightarrow} H^{0}(\mathcal{F}) \otimes \mathcal{O}_{X} \stackrel{e v}{\longrightarrow} \mathcal{F} \stackrel{\text { res }}{\longrightarrow} \mathcal{F} \otimes \mathbf{k}(z)$ is an epimorphism for any $z \in X \backslash P^{\prime}$. Moreover, since we can consider $g_{\psi}$ as an epimorphism : $V^{\vee} \otimes \mathcal{O}_{X} \rightarrow \mathcal{F}_{\mid P^{\prime}}, \varepsilon(z)$ is also an epimorphism for any $z \in P^{\prime}$. This means that $\varepsilon(z)$ is an epimorphism for any $z \in X$, 
i.e. that the morphism $\varepsilon: V^{\vee} \otimes \mathcal{O}_{X} \rightarrow \mathcal{F}$ is an epimorphism. By the universality property of $G$ this means that there exists a morphism $f=f_{\varepsilon}: X \rightarrow G$ such that $\varepsilon=f_{\varepsilon}^{*} \gamma, \mathcal{F} \simeq f_{\varepsilon}^{*} Q$, where $\gamma: V^{\vee} \otimes \mathcal{O}_{G} \rightarrow Q$ is the canonical epimorphism. This together with (69) yields (iii). In addition, since $\psi$ is determined by $g_{\psi}$, the equality $g_{\psi}=\operatorname{res}_{P^{\prime}}(\varepsilon)$ directly implies (ii).

Next, we apply the functor $\mathcal{H} m_{\mathcal{O}_{X}}\left(V^{\vee} \otimes \mathcal{O}_{X},-\right)$ to the commutative diagram

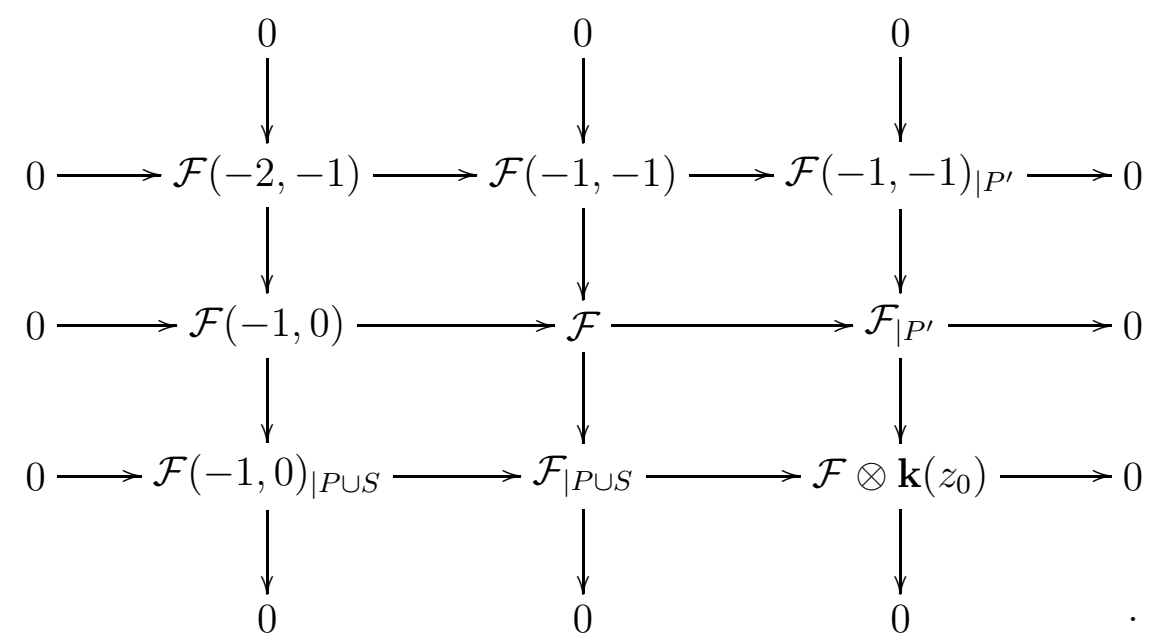

Using (63), (64), (70)-(72), we obtain the commutative diagram

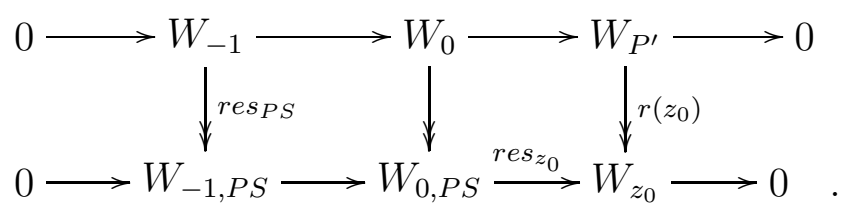

Moreover, setting $\varepsilon_{z_{0}}:=r\left(z_{0}\right)\left(g_{\psi}\right)$, we have

$$
\operatorname{res}_{z_{0}}^{-1}\left(\varepsilon_{z_{0}}\right) \simeq W_{-1, P S} .
$$

Similarly to (77), using Theorem 5.2 and (70)-(71), we obtain the surjective restriction maps

$$
\operatorname{res}_{P} W_{P} \stackrel{\operatorname{res}_{P}}{\longleftarrow} W_{-1, P S} \stackrel{\operatorname{res}_{S}}{\rightarrow} W_{-1, S} \simeq \operatorname{Hom}\left(V^{\vee}, H^{0}\left(\left(\mathcal{O}_{\mathbb{P}^{1}}(1)\right)^{r}\right)\right) .
$$

Now (77) and (78) together with Corollary 4.5 show that

$$
U:=\operatorname{res}_{P S}^{-1}\left(\left(\operatorname{res}_{P}\right)^{-1}\left(W_{P}\left(E, C_{0}\right)\right) \cap\left(\operatorname{res}_{S}\right)^{-1}\left(W^{*}\left(E, y_{0}\right)\right)\right)
$$

is a dense open subset of $W_{-1}$. Hence, for a generic element $\varepsilon \in U$ the corresponding morphism $f=f_{\varepsilon}: X \rightarrow G$ satisfies the conditions (a) $e_{\pi}:=\operatorname{res}_{P}\left(\operatorname{res}_{P S}(\varepsilon)\right) \in W_{P}\left(E, C_{0}\right)$ and (b) $e_{\rho}:=$ $\operatorname{res}_{S}\left(\operatorname{res}_{P S}(\varepsilon)\right) \in W^{*}\left(E, y_{0}\right)$. Here, by construction, we have $\varphi_{e_{\pi}}=f \circ \pi$ and, respectively, $\varphi_{e_{\rho}}=$ $f \circ \rho$. Now (a) together with (67) and (68) means that $f(P)=\operatorname{im} f \circ \pi=\operatorname{im} \varphi_{e_{\pi}} \in B_{P}\left(E, C_{0}\right)$, i.e. that $\mathbf{D}\left(E_{\mid f(P)}\right) \leq \mathbf{D}\left(E_{\mid C_{0}}\right)$; respectively, (b) together with (31) and Corollary 4.4 means that $\mathbf{D}\left(E_{\mid f(S)}\right) \leq 2 r \mathbf{D}(E)$. This yields (iv). The claim (i) is clear from the construction.

\section{MAIN RESUlT}

We now proceed to the main construction. Consider a twisted ind-Grassmannian $\mathbf{G}$ defined by (1), together with a vector bundle on $\mathbf{G}$, i.e. with vector bundles $E_{m}$ on $G_{m}:=G\left(r_{m}, V^{n_{m}}\right)$ of fixed rank such that $\varphi_{m}^{*} E_{m+1}=E_{m}$ for $m \geq 1$. We assume that $G_{m}$ is not isomorphic to a projective space for any $m$. Set

$$
\Phi_{m}:=\varphi_{m-1} \circ \ldots \circ \varphi_{1}: G_{1} \hookrightarrow G_{m} .
$$


The basic assumption (2) that $\mathbf{G}$ is sufficiently twisted can be rewritten as

$$
\lim _{m \rightarrow \infty} \frac{r_{m}}{\operatorname{deg} \Phi_{m}}=0 .
$$

Note that (79) is always satisfied if $r_{m}$ doesn't depend on $m$.

For a given integer $m>1$, fix a line $l_{0}$ in $G_{m}$ such that

$$
\mathbf{D}\left(E_{m \mid l_{0}}\right)=\max _{\text {lines } l \subset G_{m}} \mathbf{D}\left(E_{m \mid l}\right)=: \mathbf{D}_{m} .
$$

Let also $l_{1}$ be any line in $G_{1}$ such that

$$
\mathbf{D}\left(E_{1 \mid l_{1}}\right)=\max _{\text {lines } l \subset G_{1}} \mathbf{D}\left(E_{1 \mid l}\right)=: \mathbf{D}_{1} .
$$

(Clearly, such lines $l_{0}$ and $l_{1}$ exist by semicontinuity).

Put $C_{0}:=\Phi_{m}\left(l_{1}\right), \quad d:=\operatorname{deg} C_{0}=\operatorname{deg} \Phi_{m}$. Fix a point $y_{0} \in l_{0}$ and a curve $C \in B\left(E, y_{0}\right)$ (see Theorem 4.4) and fix a degree $d$ morphism $\psi: \mathbb{P}^{1} \rightarrow l_{0} \hookrightarrow G_{m}$. Consider the surface $X=\mathbb{P}^{1} \times \mathbb{P}^{1}$ together with the distinguished fibers $P, P^{\prime}$ of the projection $p r_{1}: X \rightarrow \mathbb{P}^{1}$ and $S$ of the projection $p r_{1}: X \rightarrow \mathbb{P}^{1}$. Applying Theorem 6.1 to this datum, we obtain a morphism $f: X \rightarrow G_{m}$ such that, for $E_{X}:=f^{*} E_{m}$, we have:

(i) the morphism $\mathbb{P}^{1} \stackrel{\left(p r_{2 \mid P^{\prime}}\right)^{-1}}{\sim} P^{\prime} \stackrel{f}{\rightarrow} G_{m}$ coincides with $\psi$, hence by (이) and (미) the vector bundle $E_{X}$ satisfies the equality

$$
\mathbf{D}\left(E_{X \mid P^{\prime}}\right)=\operatorname{deg}\left(f_{\mid P^{\prime}}\right) \mathbf{D}\left(E_{m \mid l_{0}}\right)=\left(\operatorname{deg} \Phi_{m}\right) \mathbf{D}_{m}
$$

(ii) $f_{\mid P}$ and $f_{\mid S}$ are embeddings such that

$$
\begin{gathered}
\mathbf{D}\left(E_{X \mid P}\right)=\mathbf{D}\left(E_{m \mid f(P)}\right) \leq \mathbf{D}\left(E_{m \mid C_{0}}\right)=\mathbf{D}\left(E_{m \mid \Phi_{m}\left(l_{1}\right)}\right)=\mathbf{D}\left(E_{1 \mid l_{1}}\right)=\mathbf{D}_{1} \\
\mathbf{D}\left(E_{X \mid S}\right)=\mathbf{D}\left(E_{m \mid f(S)}\right) \leq 2 r_{m} \mathbf{D}_{m} .
\end{gathered}
$$

Now applying the inequality (3.11) from [DP] to (82)-(84) we obtain

$$
\left(\operatorname{deg} \Phi_{m}\right) \mathbf{D}_{m}=d \mathbf{D}_{m} \leq 4 \mathrm{rk} E_{X}\left(\mathbf{D}_{1}+2\right)\left(2 r_{m} \mathbf{D}_{m}+1\right)-2 \mathrm{rk} E_{X}
$$

But this inequality clearly contradicts to (79) for large enough $m$ if $\mathbf{D}_{m} \neq 0$. Hence $\mathbf{D}_{m}=0$. Therefore, by [PT, Proposition 4.1] $E_{m}$ is trivial. We thus have proved our main result.

Theorem 7.1. There are no nontrivial vector bundles of finite rank on a sufficiently twisted ind-Grassmannian $\mathbf{G}$.

We conclude this paper by a class of natural examples of twisted ind-Grassmannians (11) with $r_{m}=r=$ const. Recall that any embedding of $\mathbb{P}^{n}$ to $\mathbb{P}^{n^{\prime}}$ is given by a subsystem of a complete linear system, i.e. by a composition of a Veronese embedding of $\mathbb{P}^{n}$ into $\mathbb{P}^{n^{\prime \prime}}, n^{\prime \prime} \geq n^{\prime}$, and subsequent projection of $\mathbb{P}^{n^{\prime \prime}}$ to $\mathbb{P}^{n^{\prime}}$. In fact, this procedure extends to Grassmannians of $r$-dimensional subspaces, $r$ being fixed. More precisely, for each $m \geq 1$ fix an integer $k_{m}>1$ and construct the Grassmannians $G_{m}=G\left(r, V_{m}\right)$ and their successive embeddings $\varphi_{m}: G_{m} \hookrightarrow G_{m+1}$ inductively by the following procedure. Consider the flag variety $\Gamma_{m}=F l\left(1, r ; V_{m}\right)$ together with the natural embedding $\Gamma_{m} \hookrightarrow P\left(V_{m}\right) \times G_{m}$ and the sheaf $\mathcal{O}_{\Gamma}\left(1, k_{m}\right):=\left(\mathcal{O}_{P\left(V_{m}\right)}(1) \otimes \mathcal{O}_{G_{m}}\left(k_{m}\right)\right)_{\mid \Gamma_{m}}$. Set $W_{m+1}:=H^{0}\left(\mathcal{O}_{\Gamma_{m}}\left(1, k_{m}\right)\right)^{\vee}$. The embedding $\theta_{m}: \Gamma_{m} \hookrightarrow P\left(W_{m+1}\right)$ by the complete linear series $\left|\mathcal{O}_{\Gamma_{m}}\left(1, k_{m}\right)\right|$ is (by construction) induced by a homogeneous embedding (in the sense of [DP, Sect. 4]) $\psi_{m}: G_{m} \hookrightarrow G\left(r, W_{m+1}\right)$. By composing $\psi_{m}$ with a possible rational projection of the form $\pi: G\left(r, W_{m+1}\right) \rightarrow G_{m+1}=G\left(r, V_{m+1}\right)$, where $V_{m+1}$ is an appropriate quotient of $W_{m+1}$, we obtain an embedding $\varphi_{m}: G_{m} \hookrightarrow G_{m+1}$.

Note that Theorem 7.1 was proved in DP for ind-Grassmannians (11) defined via certain homogeneous morphisms $\varphi_{m}$ called twisted extensions, see [DP, Sect. 4.2]. It is not difficult to check that the above contructed embeddings $\varphi_{m}$ are not twisted extensions, hence Theorem 7.1 is new for the corresponding ind-Grassmannians G. 


\section{REFERENCES}

[BV] W. Barth and A. Van de Ven, On the geometry in codimension 2 in Grassmann manifolds, Lecture Notes in Math. 412 (1974), 1-35.

[CT] I. Coanda I., G. Trautmann The splitting criterion of Kempf and the Babylonian tower theorem, arXiv:math.AG/0411636 v.1, 29 Nov 2004.

[DiP] I. Dimitrov, I. Penkov, Ind-varieties of generalized flags as homogeneous spaces for classical indgroups, IMRN 2004, No. 55, 2935-2953.

[DP] J. Donin, I. Penkov, Finite rank vector bundles on inductive limits of grassmannians, IMRN 2003, No. 34, 1871-1887.

[K] M. Kapranov, Veronese curves and Grothendieck-Knudsen moduli space $\overline{M_{0, n}}$, J. Algebraic Geometry 2 (1993), 239-262.

[PT] I. Penkov, A. S. Tikhomirov, Rank 2 vector bundles on ind-Grassmannians, Manin Festschrift, Birkhäuser 2007, to appear.

[S1] E. Sato, On the decomposability of infinitely extendable vector bundles on projective spaces and Grassmann varieties, J. Math. Kyoto Univ. 17 (1977), 127-150.

[S2] E. Sato, On infinitely extendable vector bundles on $G / P$, J. Math. Kyoto Univ. 19 (1979), 171-189.

[St] S. A. Strømme, On parametrized rational curves in Grassmann varieties, Lecture Notes in Math 1266 (1987), 251-272.

[T] A. N. Tyurin, Vector bundles of finite rank over infinite varieties, Math. USSR Izvestija, 10 (1976), 1187-1204.

Jacobs University Bremen, School of Engineering and Science, Campus Ring 1, 28759 Bremen, GERMANY

E-mail address: ivanpenkov@jacobs-university.de

Department of Mathematics, State Pedagogical University, Respublikanskaya Str. 108 150000 Yaroslavl, Russia

E-mail address: astikhomirov@mail.ru 